\title{
Hemodynamic Correlates of Fluctuations in Neuronal Excitability: A Simultaneous Paired Associative Stimulation (PAS) and functional Near Infra-Red Spectroscopy (fNIRS) Study
}

Zhengchen Cai ${ }^{* 1}$, Giovanni Pellegrino ${ }^{* 2,3}$, Amanda Spilkin ${ }^{1}$, Edouard Delaire ${ }^{1}$, Makoto Uji ${ }^{1}$, Chifaou Abdallah ${ }^{2}$, Jean-Marc Lina ${ }^{4,5}$, Shirley Fecteau ${ }^{6,7}$, Christophe Grova ${ }^{1,2,3,5}$

1 Multimodal Functional Imaging Lab, Department of Physics and PERFORM Centre, Concordia University, Montréal, Québec, Canada

${ }^{2}$ Neurology and Neurosurgery Department, Montreal Neurological Institute, McGill University, Montréal, Québec, Canada

${ }^{3}$ Multimodal Functional Imaging Lab, Biomedical Engineering Department, McGill University, Montréal, Québec, Canada

${ }^{4}$ Département de Génie Electrique, École de Technologie Supérieure, Montréal, Québec, Canada

${ }^{5}$ Centre De Recherches En Mathématiques, Montréal, Québec, Canada

${ }^{6}$ CERVO Brain Research Centre, Centre Intégré Universitaire De Santé Et De Services Sociaux De La Capitale-Nationale, Canada

${ }^{7}$ Department of Psychiatry and Neurosciences, Faculty of Medicine, Université Laval, Canada

* Both authors contributed equally to this work.

\section{Correspondence:}

Zhengchen Cai,

Multimodal Functional Imaging Lab,

Department of Physics and PERFORM Centre, Concordia University.

Loyola Campus, Office SP 365.23

7141 Sherbrooke Street West, Montreal, QC, H4B 1R6, Canada

Phone: (514) 848-2424 ext.3393

Corresponding author emails: zhengchen.cai@mail.concordia.ca; zhengchen.cai@gmail.com 


\section{Acknowledgments}

This work was supported by the Natural Sciences and Engineering Research Council of Canada

Discovery Grant Program (CG and JML), an operating grant from the Canadian Institutes for Health Research (CIHR MOP 133619 (CG)), a FRQNT research team grant and a FRQS-Quebec Bio-Imaging Network (QBIN) Pilot Project grant. fNIRS equipment was acquired using grants from NSERC Research Tools and Instrumentation Program and the Canadian Foundation for Innovation (CG). SF is supported by the Canada Research Chair in Cognitive Neuroplasticity. ZC is funded by the Fonds de recherche du Québec - Sante (FRQS) Doctoral Training Scholarship and the PERFORM Graduate Scholarship in Preventive Health Research. GP is funded by Strauss Canada Foundation and a McGill/MNI - Fred Andermann EEG and Epilepsy Fellowship. MU is funded by the Horizon Postdoctoral Fellowships of Concordia University. 


\begin{abstract}
Background: The relationship between task-related hemodynamic activity and brain excitability is poorly understood in humans as it is technically challenging to combine simultaneously noninvasive brain stimulation and neuroimaging modalities. Cortical excitability corresponds to the readiness to become active and as such it may be linked to metabolic demand.
\end{abstract}

Hypotheses: Cortical excitability and hemodynamic activity are positively linked so that increases in hemodynamic activity correspond to increases in excitability and vice-versa.

Methods: Fluctuations of excitability and hemodynamic activity were investigated via simultaneous Transcranial Magnetic Stimulation (TMS) and functional Near Infrared Spectroscopy (fNIRS). Sixteen healthy subjects participated in a sham-controlled, pseudorandomized, counterbalanced study with PAS (PAS10/PAS25/Sham) on the right primary motor cortex (M1). The relationship between M1 excitability (Motor Evoked Potentials, MEP) and hemodynamic responses to finger tapping reconstructed via personalized fNIRS was assessed.

Results: Hemodynamic activity exhibited a significant correlation with cortical excitability: increased $\mathrm{HbO}$ and $\mathrm{HbR}$ (absolute amplitude) corresponded to increased excitability and viceversa $(r=0.25 ; p=0.03$ and $r=0.16 ; p=0.17$, respectively). The effect of PAS on excitability and hemodynamic activity showed a trend of positive correlation: correlation of MEP ratios (postPAS/pre-PAS) with $\mathrm{HbO}$ and $\mathrm{HbR}$ ratios ( $\mathrm{r}=0.19, \mathrm{p}=0.29 ; \mathrm{r}=0.18, \mathrm{p}=0.30$, respectively).

Conclusions: TMS-fNIRS is a suitable technique for simultaneous investigation of excitability and hemodynamic responses and indicates a relationship between these two cortical properties. PAS effect is not limited to cortical excitability but also impacts hemodynamic processes. These findings have an impact on the application of neuromodulatory interventions in patients with neuropsychiatric disorders.

Keywords: Paired Associative Stimulation (PAS), Excitability, Maximum Entropy on the Mean (MEM), Finger Tapping, functional Near Infrared Spectroscopy (fNIRS), Transcranial Magnetic Stimulation (TMS) 


\section{Introduction}

The relationship between cortical excitability and elicited hemodynamic activity is poorly understood in humans. Such investigation requires simultaneous measurements of excitability and hemodynamic activity [Siebner et al., 2009]. Transcranial Magnetic Stimulation (TMS) is a versatile technique that allows the assessment and modulation of primary motor cortex (M1) excitability. Cortical excitability assessment is easily achieved via single pulse TMS (spTMS) by measuring the amplitude of the Motor Evoked Potentials (MEPs). M1 excitability modulation can be obtained by repetitive TMS (rTMS) and related techniques, such as Paired Associative Stimulation (PAS). PAS was established about two decades ago [Stefan, 2000] and consists in combining TMS with peripheral electrical stimulation such as Median Nerve Stimulation (MNS). It relies on the principle that repetitive stimulations delivered with proper timing and pace induce long-term potentiation and long-term depression like plasticity, exploiting the concept of spiketiming-dependent plasticity [Levy and Steward, 1983; Rossini et al., 2015]. PAS effects can last for 30 minutes or more [Lee et al., 2017; Stefan, 2000; Suppa et al., 2017]. This technique is therefore well-suited to manipulate cortical excitability by tuning the timing of the interstimulus intervals (ISI), because it can generate either an excitability increase, when applying a $25 \mathrm{~ms}$ time-interval between MNS and TMS pulses (PAS25), or an excitability decrease, when applying a 10ms time-interval between pulses (PAS10). A PAS sham has also been described and validated [Gow et al., 2004; Loo et al., 2000; Michou et al., 2014].

A standard approach to non-invasively map brain functions is to measure the fluctuations of brain hemodynamic signals elicited by neuronal activity. Functional magnetic resonance imaging (fMRI) is typically the tool of choice for its reliability, ease of use, high spatial resolution and sensitivity to deep brain regions [Bandettini et al., 1992; Glover, 2011; Kwong et al., 1992]. Nevertheless, bringing TMS within the MRI environment is challenging [Hallett et al., 2017]. MRI compatible TMS coils have been developed [Navarro De Lara et al., 2015; Wang et al., 2017] and applied to investigate the TMS induced hemodynamic responses [Navarro de Lara et al., 2017], but the majority of studies have had an "offline" approach, meaning that no continuous fMRI measurement during TMS is usually performed [Siebner et al., 2009].

Functional Near Infra-Red Spectroscopy (fNIRS) is a non-invasive neuroimaging modality, which allows monitoring changes in oxy- and deoxy-hemoglobin (i.e., $\mathrm{HbO} / \mathrm{HbR}$ ) in the cerebral 
cortex [Jöbsis, 1977; Scholkmann et al., 2014; Yücel et al., 2021]. It measures light intensity changes, modulated by local absorption associated with underlying hemoglobin concentration fluctuations, via source-detector pairs placed on the scalp. fNIRS has high temporal resolution and acceptable spatial resolution with no electromagnetic interference [Curtin et al., 2019b; Siebner et al., 2009; Vasta et al., 2017]. It allows the estimation of both $\mathrm{HbO}$ and $\mathrm{HbR}$ concentration changes in cortical regions. It is relatively portable and permits prolonged scans, with little or no discomfort for the participants [Gramigna et al., 2017; Pellegrino et al., 2016; Scholkmann et al., 2014]. The drawback of combining fNIRS and TMS is that when both techniques are applied to the same cortical area, the optodes (fNIRS sensors) introduce some additional space between the TMS coil and the scalp. This requires higher TMS stimulation intensities as the strength of the magnetic field decays sharply when increasing the coil distance to the target region [Curtin et al., 2019b; Parks, 2013]. Nevertheless, we are proposing combining PAS and fNIRS as a promising way to assess the relationship between cortical excitability and hemodynamic responses.

We conducted here the first PAS-fNIRS investigations of the relationship between cortical excitability and task-related hemodynamic response. Cortical excitability corresponds to the cortical readiness to become active and as such it may be linked to metabolic demand. We first hypothesized that fluctuations of cortical excitability are positively correlated with fluctuations of hemodynamic activity. This hypothesis was tested by estimating the correlation between MEP peak-peak amplitude and task-related $\mathrm{HbO} / \mathrm{HbR}$ response, regardless of the PAS interventions (PAS25, PAS10, Sham). Second, we further hypothesized that PAS affects cortical task-related hemodynamic responses in addition to cortical excitability, and such modulations of excitability and hemodynamic positively correlate. In other words, when PAS increases cortical excitability, it results in enhanced task-related hemodynamic response, whereas when PAS decreases cortical excitability, it results in reductions of the hemodynamic response to the task. This hypothesis was tested by estimating the correlation between $\mathrm{MEP}$ and $\mathrm{HbO} / \mathrm{HbR}$ ratios calculated as the post- over pre-intervention amplitudes.

\section{Material and methods}

\subsection{Subjects and study design}


This study was approved by the Central Committee of Research Ethics of the Minister of Health and Social Services Research Ethics Board, (CCER), Québec, Canada. All subjects signed a written informed consent before participation. Only subjects meeting the following inclusion/exclusion criteria were considered: 1) age between 18 and 40 years old; 2) righthanded male; 3) no present or past neurological disorders; 4) no medications acting on the central nervous system; 5) no contraindications to MRI or TMS [Rossi et al., 2009; Suppa et al., 2017]. Nineteen subjects (19 - 35 years old) participated in the study. Subjects were instructed to have a regular sleep cycle for the days before the experiments and to not consume caffeine for at least 90 minutes before the experiment.

The experimental design and setup are illustrated in Fig.1 and Fig.2, respectively. Every participant had 1) an anatomical head MRI scan (T1- and T2-weighted, $1 \mathrm{~mm}^{3}$ isotropic) for neuronavigated TMS; calculate personalized fNIRS optical head model [Machado et al., 2014; Machado et al., 2018]; and install optodes, followed by 2) somatosensory evoked potentials recording during electrical stimulation of the median nerve at the wrist to measure N20 latency and tune PAS accordingly. We then conducted three experimental sessions corresponding to three different PAS interventions: PAS25, PAS10, and sham. These sessions were performed at least two days apart to minimize potential carryover effects. As this study included three sessions, we decided to consider male participants to minimize the confounding of cortical excitability changes due to the menstrual cycle [Hattemer et al., 2007; Lee et al., 2017]. Experimental sessions were performed with a pseudorandomized order, counterbalanced across subjects, which consisted of the following components:

- $\quad$ PAS was performed with 100 pairs of MNS and TMS with a fixed interval of 10s according to the guidelines [Suppa et al., 2017], for a total duration of 18 minutes (Fig.1c). MNS was delivered at the left wrist and with the following parameters: intensity $=300 \%$ perceptual threshold, square wave and $0.2 \mathrm{~ms}$ duration. TMS intensity was $120 \%$ of the resting motor threshold (RMT). The ISI between MNS and TMS was then set to be individual N20+5ms for PAS25 and N20-5ms for PAS10, respectively [Carson and Kennedy, 2013]. Sham was the same as PAS25, except TMS was not delivered, whereas its sound was provided via a stereo speaker [Zangrandi et al., 2019].

Before and after each intervention, 
- Cortical excitability was measured via M1 spTMS, applying 75 stimuli (ISI ranging between 5s and 25s) to the right hand-knob (Fig.1b). MEPs were recorded by bipolar electromyography (EMG) electrodes attached on the right abductor pollicis brevis (APB), with a standard belly-tendon montage (Fig.2d). The RMT was defined with the TMS Motor Threshold Assessment Tool [Ah Sen et al., 2017; Awiszus et al., 1999]. All TMS procedures followed the recommendations of the International Federation of Clinical Neurophysiology, and no participants reported any severe discomfort or side effects [Rossi et al., 2009].

- A finger tapping task was performed to activate M1 and estimate its task-related hemodynamic activity. Subjects were instructed to tap the left thumb to the other digits sequentially, at a pace of about $2 \mathrm{~Hz}$ (Fig.1a). The movement was performed in short blocks of 10 s interleaved with a resting period jittered between 30 s to $60 \mathrm{~s}$. This time-constraint was meant to avoid task events phase locking to undergoing physiological hemodynamic oscillations [Aarabi et al., 2017]. Movement onset and offset were instructed by auditory cues. The duration of the motor task was about 18 minutes and consisted of 20 blocks.

fNIRS data were acquired using a Brainsight fNIRS machine (Rogue-Research Inc, Montreal Canada), sampling at $10 \mathrm{~Hz}$. MEPs were recorded by a BrainAmp ExG bipolar system (Brain Products GmbH, Germany). Please refer to Appendix A for further detailed experiment protocol. From the nineteen subjects, one was excluded due to low sensitivity to TMS and two due to poor fNIRS signal quality. Four subjects dropped out after the first session due to personal reasons, resulting in 16 PAS25, 12 PAS10 and 12 sham sessions.

\subsection{Personalized fNIRS using the optimal montage}

In order to maximize the sensitivity of fNIRS channel layout (i.e., montage) to M1 hemodynamic activity, we applied a personalized optimal montage, previously developed and validated by our group. Specifically, this montage maximizes fNIRS sensitivity along the cortical surface with good spatial overlap between channels [Cai et al., 2021b; Machado et al., 2014; Machado et al., 2018; Machado et al., 2021; Pellegrino et al., 2016]. The T1-/T2-weighted images were processed by FreeSurfer 6.0 to segment the head (i.e., scalp, skull, cerebrospinal fluid, gray matter and white matter) and generate a mid-cortical surface (i.e., a middle layer of the gray matter, between pia mater and gray-white matter interface) [Fischl et al., 2002]. The target area for the optimal montage (see Fig.2a) corresponded to the right hand-knob and was manually 
defined for each participant along the mid-surface [Raffin et al., 2015]. The personalized optimal montage was estimated imposing the following constraints: 1) 3 light sources and 15 detectors (see Fig.2b); 2) distance between source-detector pairs ranging from $2.0 \mathrm{~cm}$ to $4.5 \mathrm{~cm}$ and 3 ) large spatial overlap between channels, e.g., each source must construct at least 13 channels among 15 detectors. The output of the optimal montage procedure is a set of fNIRS optode positions along the scalp to probe the right hand-knob with the highest sensitivity. Finally, a proximity detector for recording physiological hemodynamics of the scalp was added at the center of the 3 sources [Gregg et al., 2010; Zeff et al., 2007].
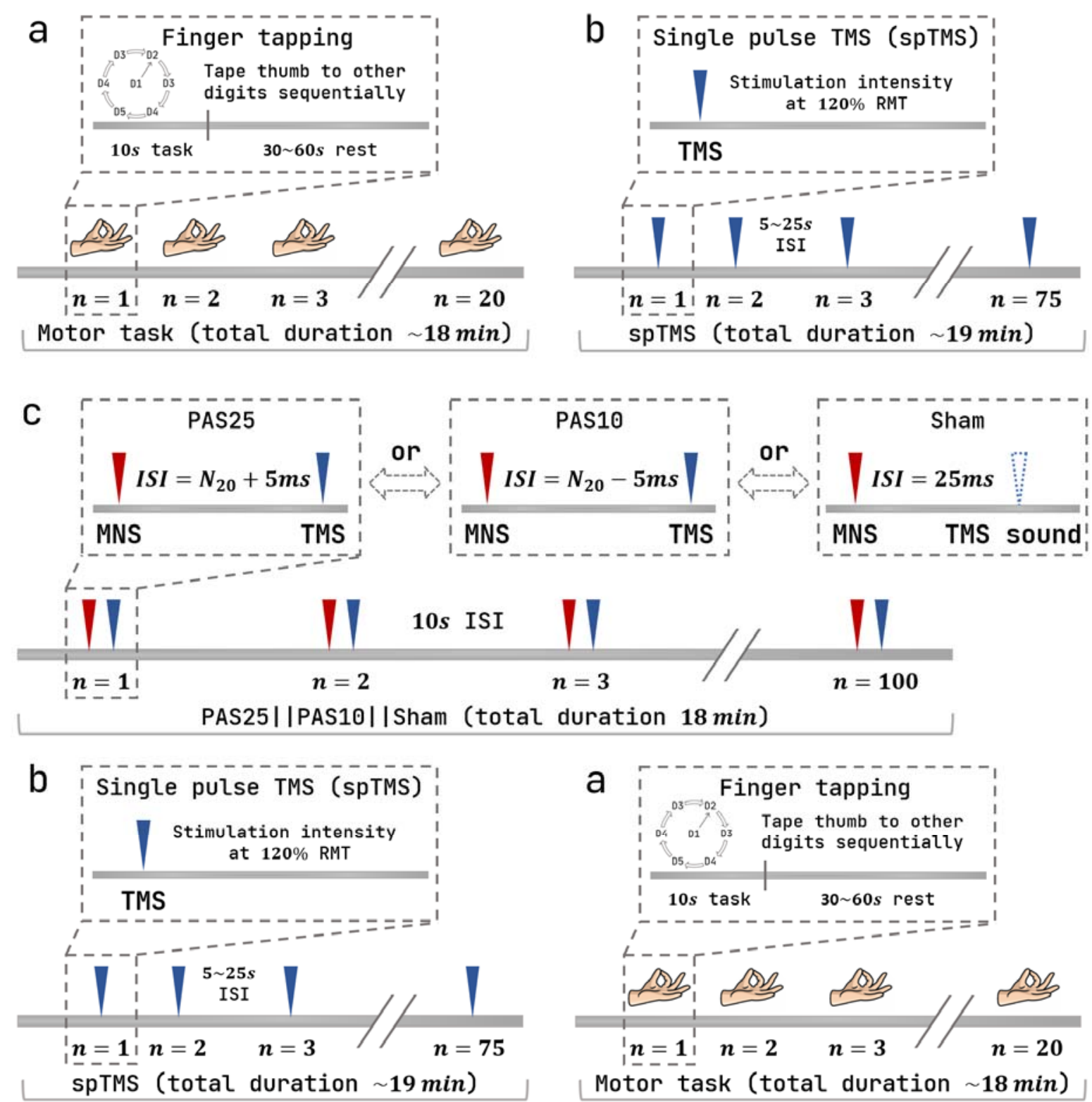
Fig.1 Study design. Each acquisition session described above contains sections following the order of a, b, c and b, a. (a) Finger tapping task: subjects were asked to tap the left thumb (D1) to the other four left digits (D2-D5) sequentially, at around 2Hz. Each tapping block lasted 10s and was followed by 30s to 60 s jitter rest. Start and stop signals were delivered by auditory cues. 20 blocks were performed for 18 minutes total duration. (b) A single pulse TMS was delivered onto the "hot spot" at $120 \%$ of individual RMT. 75 pulses were delivered with a $5 \mathrm{~s}$ to $25 \mathrm{~s}$ jittered ISI for a total duration of about 19 minutes. (c) Three PAS interventions were performed on different days separated by at least 2 days and presented in a pseudorandomized order. ISI between peripheral (MNS) and TMS was set to individual N20+5ms (PAS25) and N20-5ms (PAS10) for excitatory and inhibitory PAS, respectively. Sham was similar to PAS25, same MNS followed by a 0 intensity TMS, whereas the TMS click was reproduced via speakers. MNS intensity was set to 3x individual perceptual threshold. PAS pairs were separated by a 10s interval. In total, 100 pairs were delivered in 18 minutes. Note that fNIRS signal was acquired during the whole experiment session. 

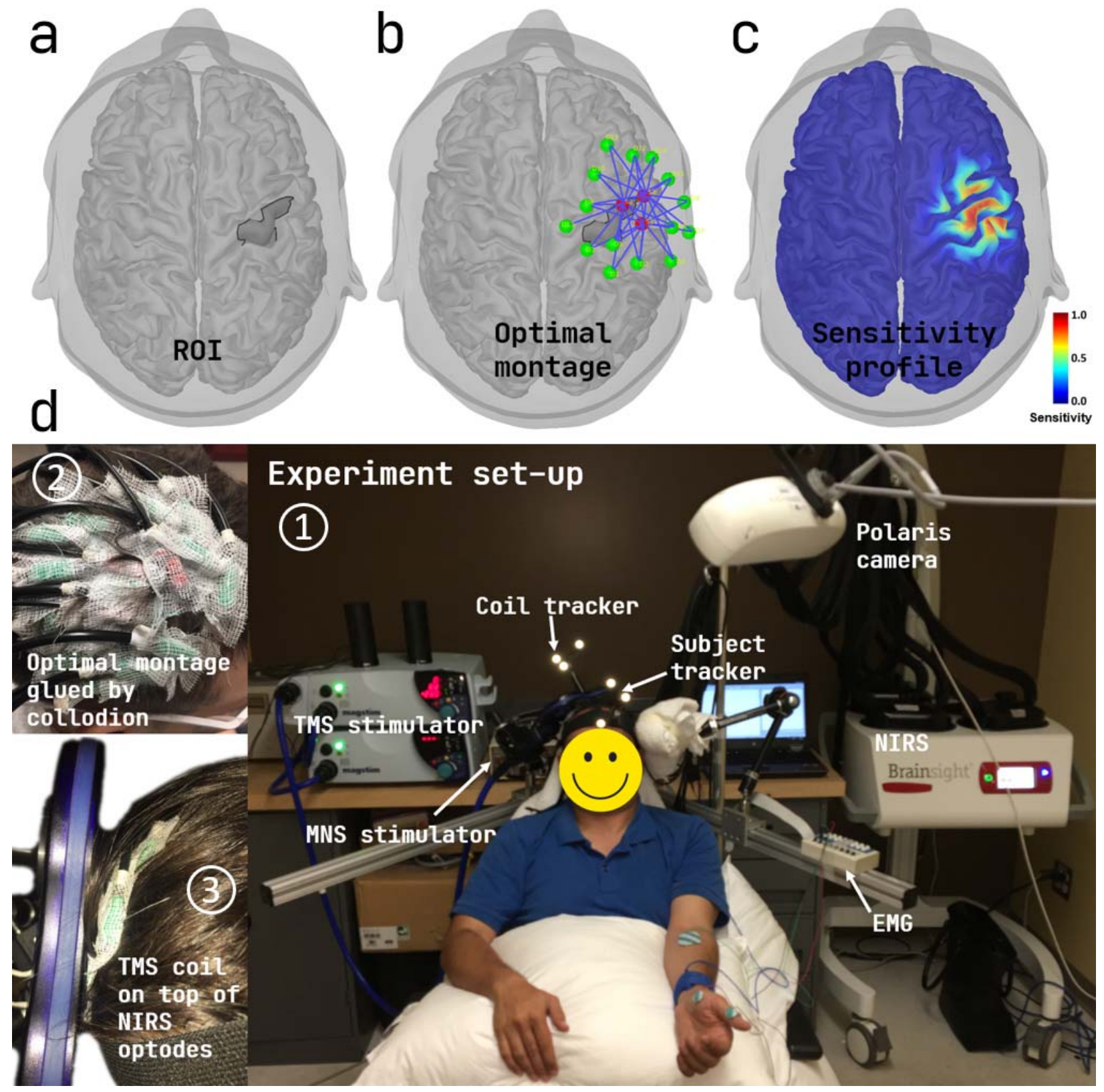

Fig.2 Personalized optimal montage and simultaneous PAS-fNIRS acquisition setup. (a) The right M1 hand region ("hand knob") was pre-defined as the target ROI of personalized optimal fNIRS montage. This area was manually selected on each subject's cortical surface extracted from individual MRI. (b) Example of the optimal montage estimated for Sub06. The montage was constrained to have 3 sources (red), 15 detectors (green) and 1 proximity detector (placed in the middle of sources, not shown). (c) Normalized light sensitivity profile of the optimal montage expressed as the sum of all channels' sensitivity along the individual cortical surface. (d) PAS and fNIRS acquisition setup. (1) Participants sat on a comfortable TMS armchair. Four different machines were involved: a Brainsight-fNIRS for fNIRS data acquisition and neuronavigation; Magstim $200^{2}$ TMS stimulators for spTMS and PAS; a Digitimer 
DS7A for MNS and PAS; and a BrainAmp ExG bipolar system for recording MEPs. (2) Low-profile (thin) optodes were attached on the subject's head using collodion based on their optimal positions defined in (b). (3) A figure-8 TMS coil was placed on top of the optodes, guided with the neuronavigation system, held with a mechanical arm.

\subsection{Excitability data analysis}

EMG data collected during spTMS were analyzed using the Brainstorm software [Tadel et al., 2011] and R 4.0.3 [R Core Team, 2020]. They were filtered between 3 and 2000Hz. MEP trials were extracted within a time window from $-10 \mathrm{~ms}$ to $100 \mathrm{~ms}$ around the stimulation and baseline corrected (-10ms to 0ms). Run specific (e.g., pre-PAS 25 of Sub01) excitability was expressed as the average of MEP peak-peak amplitudes across spTMS. Session specific (e.g., PAS 25 of Sub01) excitability change was measured as the post-/pre-PAS ratio of averaged MEP peak-peak amplitudes.

\section{4 fNIRS data processing}

The details of the following procedures are provided in Appendix B. Briefly, fNIRS data processing was performed applying 3D reconstructions with the Maximum Entropy on the Mean (MEM) framework, as described in [Cai et al., 2021a; Cai et al., 2021b]. The goal was to extract $\mathrm{HbO} / \mathrm{HbR}$ amplitude from spatiotemporal maps reconstructed from the channel space data to the underlying cortical surface. fNIRS data pre-processing involved the following steps: bad channel rejections; physiological noise regression using proximity channels [Gregg et al., 2010; Zeff et al., 2007]; band-pass filter between $0.01 \mathrm{~Hz}$ and $0.1 \mathrm{~Hz}$, and epochs extraction with a time window of -10 s to 30 s around the task onset. To extract robust and reliable $\mathrm{HbO} / \mathrm{HbR}$ amplitude for further estimation of PAS effects on hemodynamic, we introduced in this study an original approach which comprises three steps: 1) selection of 101 trials centred around the median signal to noise ratio (SNR) of "all the possible" sub-averaged 16 out of $20 \mathrm{fNIRS}$ epochs. This procedure aimed to exclude eventual motion artifacts contaminated epochs from sub-averaging and resulted in a distribution representing the variability of task-evoked fNIRS signal changes specific for each run (e.g., pre-PAS25 of Sub01); 2) fNIRS 3D reconstructions of each subaveraged trial using the MEM framework, resulted in 101 spatiotemporal hemodynamic responses maps for each task run; 3) extraction/identification of a data-driven ROI which exhibited significant task-related hemodynamic responses for each session (e.g., PAS25 of 
Sub01). Importantly, since this procedure was data-driven, fNIRS analysis was blind to PAS interventions. Finally, the measure of $\mathrm{HbO} / \mathrm{HbR}$ was defined as the averaged amplitude within a $5 \mathrm{~s}$ window centred around the peak of the reconstructed time course, extracted by averaging reconstructed time courses within the ROI defined in 3). Therefore, $101 \mathrm{HbO} / \mathrm{HbR}$ amplitudes were extracted for each run. Run specific hemodynamic responses were expressed as the averaged $\mathrm{HbO} / \mathrm{HbR}$ amplitude among all 101 trials. Session specific PAS effects on task-related hemodynamic response were represented by the post-/pre-intervention ratios of averaged $\mathrm{HbO} / \mathrm{HbR}$ measures. To demonstrate group-level intervention effects on reconstructed $\mathrm{HbO} / \mathrm{HbR}$ maps, individual maps selected at their respective peak were first coregistered on the mid-surface of MNI ICBM152 [Fonov et al., 2009; Fonov et al., 2011] template using FreeSurfer spherical registration, and then averaged over all subjects.

\subsection{Statistical analysis}

To assess the relationship between fluctuations of cortical excitability and task-related hemodynamic activity regardless of PAS intervention, we pooled together subjects' data of all runs (before and after PAS) and calculated Pearson's correlations (r) between MEPs amplitude and $\mathrm{HbO}$ responses (HbR, respectively). Furthermore, as cortical excitability is usually estimated on 20 or fewer MEPs while we had 75 measures, we applied a procedure to keep within-subject variability, that would have been lost by collapsing all trials in a single average. We then performed a bootstrap so that 2000 correlations were computed considering averaged amplitudes of $20 \mathrm{MEPs}, 20 \mathrm{HbO}$ and $20 \mathrm{HbR}$ trials randomly selected for each run. The resulting r empirical distribution allowed the estimation of an average r-value and a confidence interval.

To estimate the effect of PAS on excitability (MEPs), HbO and HbR responses, we considered post-/pre- ratios. The effects of PAS across interventions were tested with a one-way ANOVA applied independently for MEPs, $\mathrm{HbO}$ and $\mathrm{HbR}$ ratios, whereas the effect of each intervention was tested with a one-sample t-test against 1.

To estimate the relationship between PAS-related excitability and hemodynamic changes, linear regressions were performed between $\mathrm{MEP}$ and $\mathrm{HbO}$ ratios ( $\mathrm{HbR}$, respectively). The regression was conducted on three classes: 1) pooling all sessions; 2) sessions exhibiting concordant effects only, defined as MEP and $\mathrm{HbO}$ and $\mathrm{HbR}$ ratios simultaneously larger or smaller than 1; and 3) sessions with discordant effects. 


\subsection{Data and code availability}

The original raw data supporting the findings of this study are available upon reasonable request to the corresponding authors. fNIRS and TMS data were processed via Brainstorm software [Tadel et al., 2011] available at https://neuroimage.usc.edu/brainstorm/ and the fNIRS processing plugin - NIRSTORM (https://github.com/Nirstorm/nirstorm) in Brainstorm.

\section{Results}

\subsection{Correlation between cortical excitability and task-related hemodynamic responses}

Fig.3 illustrates the relationship between excitability (MEPs amplitude) and task-related hemodynamic activity ( $\mathrm{HbO}$ and $\mathrm{HbR}$ ). We found a significant positive linear correlation between MEP and $\mathrm{HbO}$ amplitude $(\mathrm{r}=0.25, \mathrm{p}=0.03)$ and a non-significant negative linear relationship between MEP amplitude and $\operatorname{HbR}(\mathrm{r}=-0.16, \mathrm{p}=0.171)$, meaning that an increased level of excitability corresponded to higher task-related $\mathrm{HbO}$ concentration and lower $\mathrm{HbR}$ concentration. These relationships were robust when considering the variability of the three amplitudes, as demonstrated by the bootstrapped correlation histograms (Fig.3c and d). The 95\% confident interval of correlation value was [0.16, 0.33] for $\mathrm{HbO}$ and $[-0.24,-0.08]$ for $\mathrm{HbR}$. In both cases, the confidence interval did not cross the zero line, meaning that the variability of excitability and hemodynamic activity measures within subjects and across trials did not influence the sign of the correlation. 

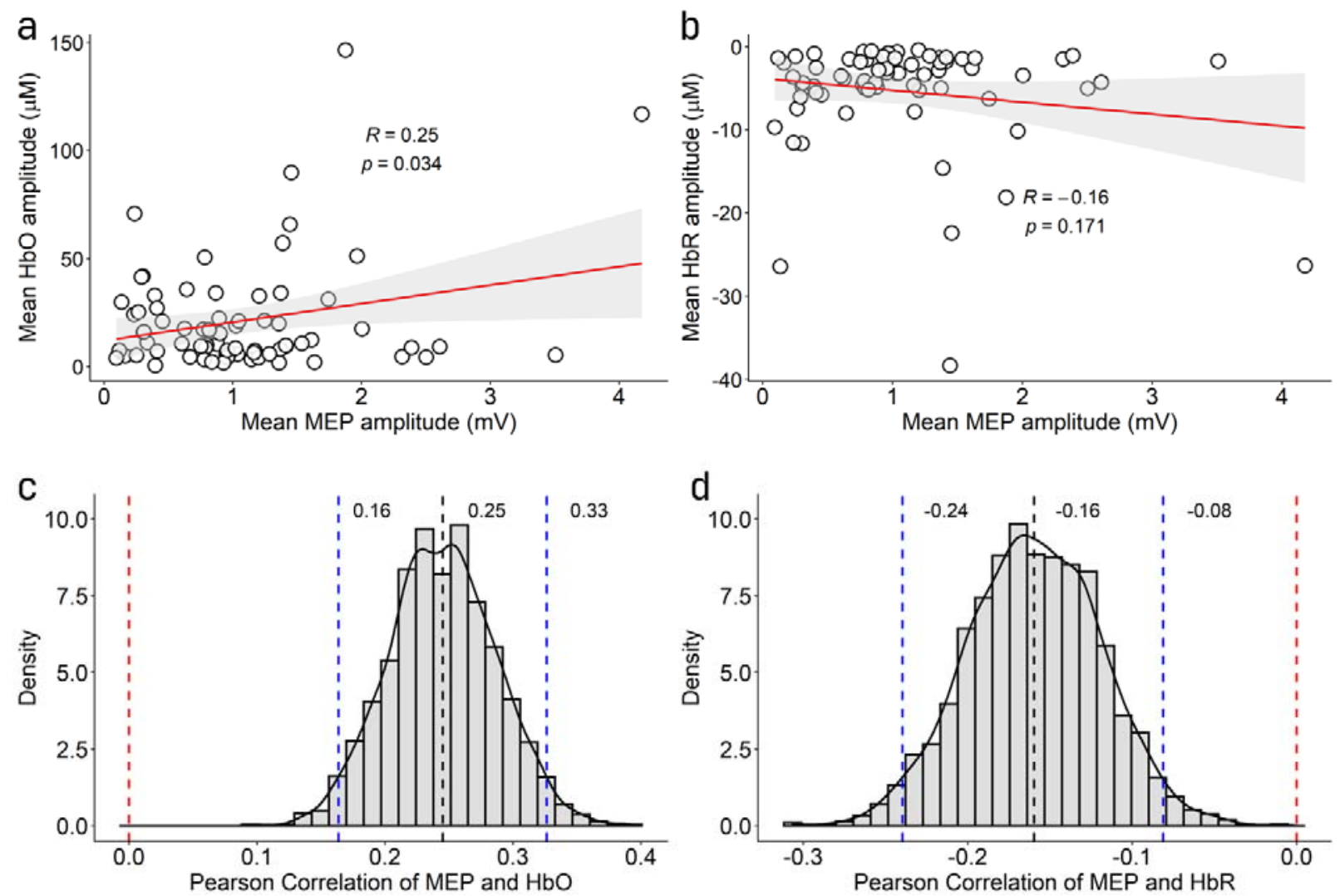

Fig.3 Correlation between MEP peak-peak amplitude and task-related HbO/HbR amplitudes. (a) scatterplot of $\mathrm{HbO}$ amplitude as a function of MEP amplitude. Each dot corresponds to a run (average over all MEPs amplitudes and all $\mathrm{HbO}$ trials). There was a significant positive linear relationship (Pearson's $r=0.25, \mathrm{p}=0.03$ ). Red lines represent the estimated regression line, and the gray area indicated the 95\% confidence interval. (b) There was a negative linear relationship between HbR and MEPs amplitude which did not reach statistical significance when considering run averages of all MEPs and $\mathrm{HbR}$ amplitudes. (c) and (d) the histogram of the correlation between MEP and $\mathrm{HbO} / \mathrm{HbR}$ amplitudes, respectively, estimated from the bootstrap procedure (selecting 20 out of $75 \mathrm{MEP}, \mathrm{HbO}$ and $\mathrm{HbR}$ trials). Black curves represented the estimated density functions; black dashed lines showed the resulting mean correlation value; blue dashed lines indicated the $95 \%$ confidence interval estimated from the histograms; red dashed lines indicated $\mathrm{r}=0$.

\subsection{PAS effects on cortical excitability and task-related hemodynamic activity}

Fig.4 shows the group-level MEP, $\mathrm{HbO}$ and $\mathrm{HbR}$ ratios for the PAS25, PAS10 and sham sessions. Overall, PAS produced similar effects for excitability and hemodynamic, with an overall increase of MEP, $\mathrm{HbO}$ and $\mathrm{HbR}$ ratios after PAS25, a decrease of MEP and $\mathrm{HbO}$ ratios after PAS10, and the ratio of sham was always in between PAS25 and PAS10. Table.1 
summarizes the corresponding ratio values. Of note, all measures and especially fNIRS measures showed rather high variabilities, which likely prevented from reaching statistical significance ( $p>0.05$ for the ANOVA and one-sample t-test) for the planned comparisons.

Fig.5 illustrates the single subject level (Sub02) PAS effects on reconstructed $\mathrm{HbO}$ and $\mathrm{HbR}$. Following PAS25 and PAS10, absolute $\mathrm{HbO}$ and $\mathrm{HbR}$ amplitude were exhibiting increases and decreases, respectively. These effects were concordant with MEP amplitude changes (detailed values in Fig.5 caption). Fig.6 presented the group-level reconstruction maps. HbO peak amplitude (mean within the ROI, ) increased after PAS25 and decreased after PAS10. HbR peak amplitude within the ROI decreased after PAS25 and also decreased after PAS10 (detailed values in Fig.6).

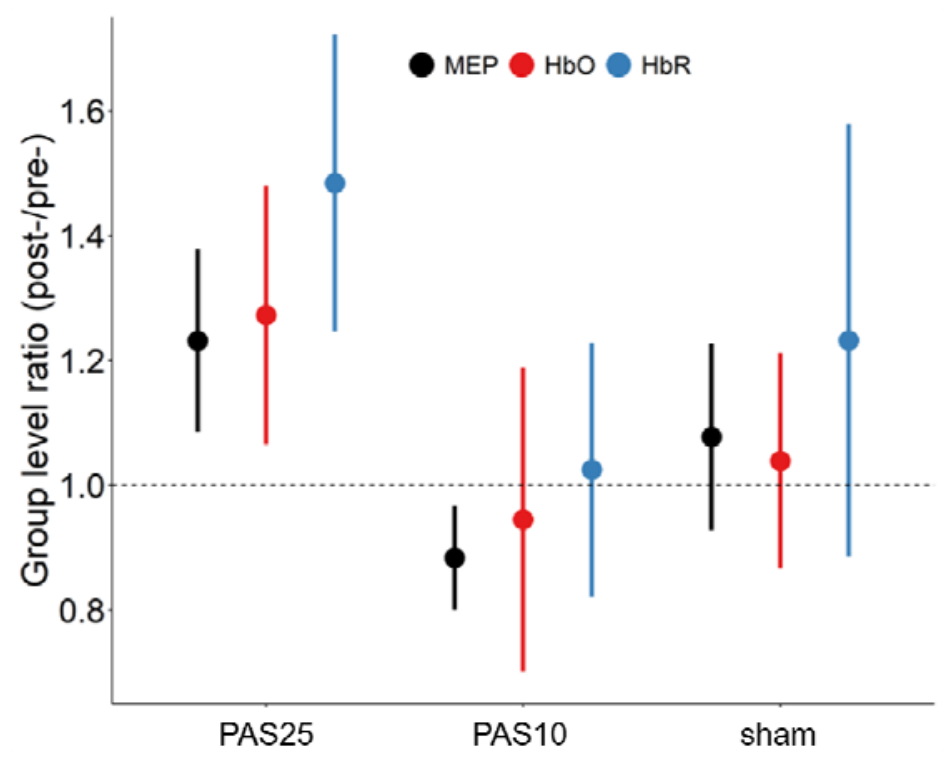

Fig.4 Group-level PAS effects on cortical excitability and hemodynamic activity. Effects of PAS25, PAS10 and sham on M1 cortical excitability (MEP amplitude) and M1 task-related hemodynamic activity ( $\mathrm{HbO}$ and $\mathrm{HbR}$ ) expressed as mean $\pm \mathrm{SEM}$ (standard error of the mean). PAS produced similar effects for excitability and hemodynamic activity, with an increase of MEP amplitude, $\mathrm{HbO}$ and $\mathrm{HbR}$ following PAS25, and a decrease of MEP amplitude and HbO following PAS10, a slight increase of the three measures after sham. All measures, and especially fNIRS measures, showed rather high variabilities (see standard error of the mean in the figure). 

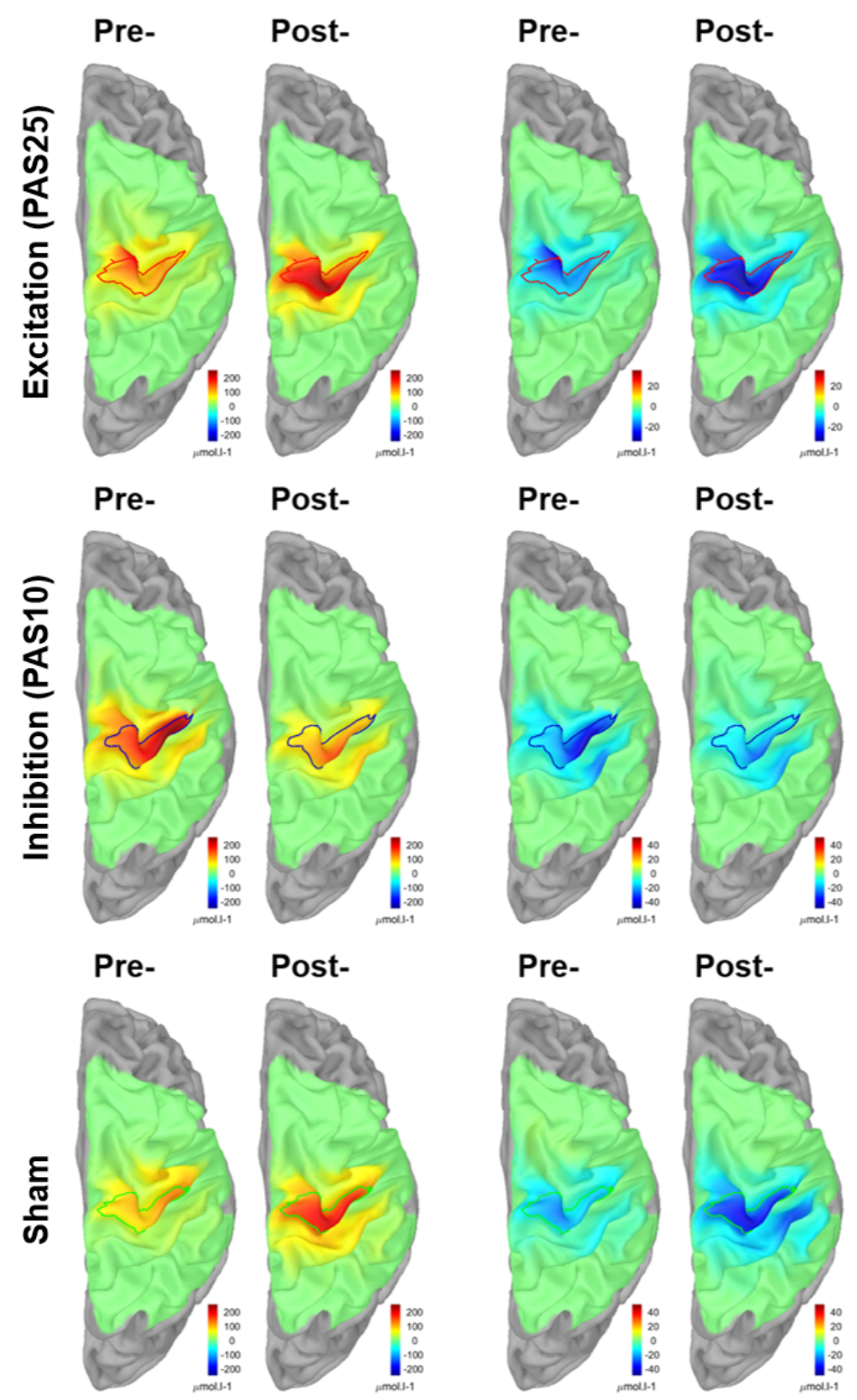

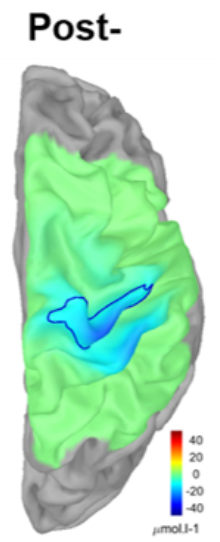

Post-

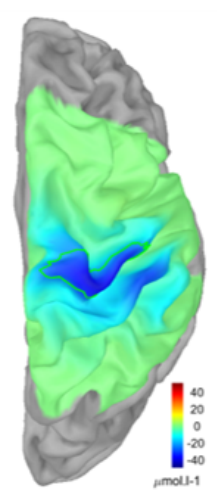

Mean of Hb time course within "hand knob"

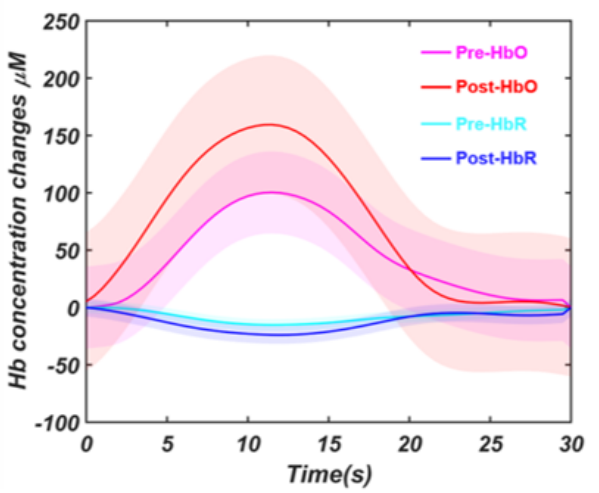

Mean of $\mathrm{Hb}$ time course within "hand knob"

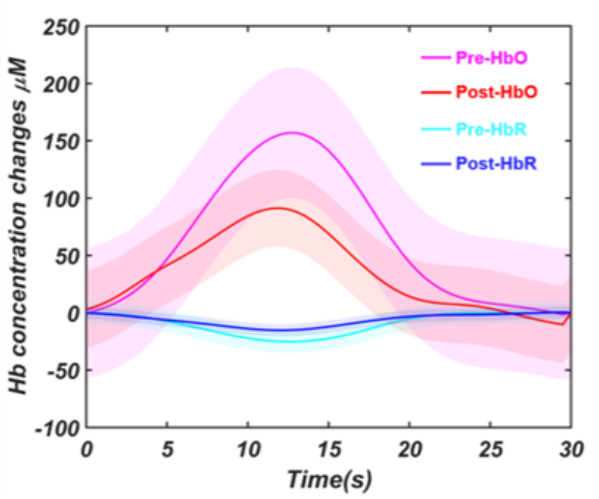

Mean of $\mathrm{Hb}$ time course within "hand knob"

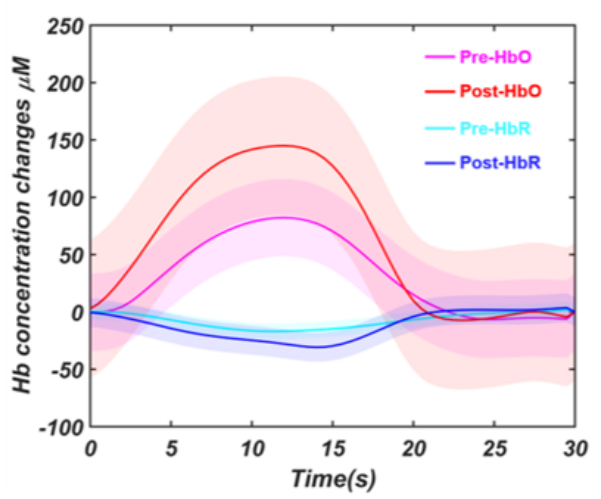

Fig.5 PAS effects on task-related hemodynamic responses using personalized fNIRS tomography

for Sub02. Individual-level reconstructed fNIRS maps of $\mathrm{HbO}$ and $\mathrm{HbR}$ responses for each experimental session, pre- or post-intervention, PAS25 ( $1^{\text {st }}$ row), PAS10 ( $2^{\text {nd }}$ row), sham ( $3^{\text {rd }}$ row). Each map demonstrated the spatial distribution pattern of the task-related hemodynamic responses at its own peak timing of the reconstructed time courses showed in the last column. The red, blue and green profiles represented the extracted specific ROIs exhibiting significant hemodynamic responses at the peak amplitudes (see method section for details) for PAS25, PAS10 and sham, respectively. The averaged time course of $\mathrm{HbO}$ and $\mathrm{HbR}$ within each selected $\mathrm{ROI}$ for each session is presented in the last column by the 
solid lines, within a time window from 0s (the task onset) to $30 \mathrm{~s}$. All $\mathrm{HbO}$ and $\mathrm{HbR}$ time courses demonstrated the typical hemodynamic responses evoked by a 10 s duration task. The shaded area represented the standard deviations of the time course within the ROI. Both the absolute amplitudes of $\mathrm{HbO}$ and $\mathrm{HbR}$ showed expected increase (after PAS25) and decrease (after PAS10) patterns within the selected ROIs. However, sham session also resulted in increased $\mathrm{HbO}$ and decreased $\mathrm{HbR}$. The corresponding ratios of $\mathrm{HbO}$ and $\mathrm{HbR}$ were also in agreement with the mean MEP ratios, e.g., MEP ratio $=1.37, \mathrm{HbO}$ ratio $=1.62$ and $\mathrm{HbR}$ ratio $=1.56$ for PAS25; then $0.85,0.58$ and 0.61 for PAS10, respectively. Colour maps for pre- and post- maps were fixed for specific hemoglobin and session (e.g., $\mathrm{HbO}$ in PAS25). $\mathrm{HbO}$ amplitudes generally exhibit a larger range than $\mathrm{HbR}$ amplitudes.

Table.1 Group-level PAS effects on cortical excitability and hemodynamic activity.

\begin{tabular}{cccc}
\hline & \multicolumn{3}{c}{ Ratio (Mean \pm SEM) } \\
& PAS25 & PAS10 & Sham \\
\hline MEP & $1.23 \pm 0.15$ & $0.88 \pm 0.08$ & $1.08 \pm 0.15$ \\
HbO & $1.27 \pm 0.21$ & $0.95 \pm 0.24$ & $1.04 \pm 0.17$ \\
HbR & $1.48 \pm 0.24$ & $1.02 \pm 0.20$ & $1.23 \pm 0.35$ \\
\hline
\end{tabular}




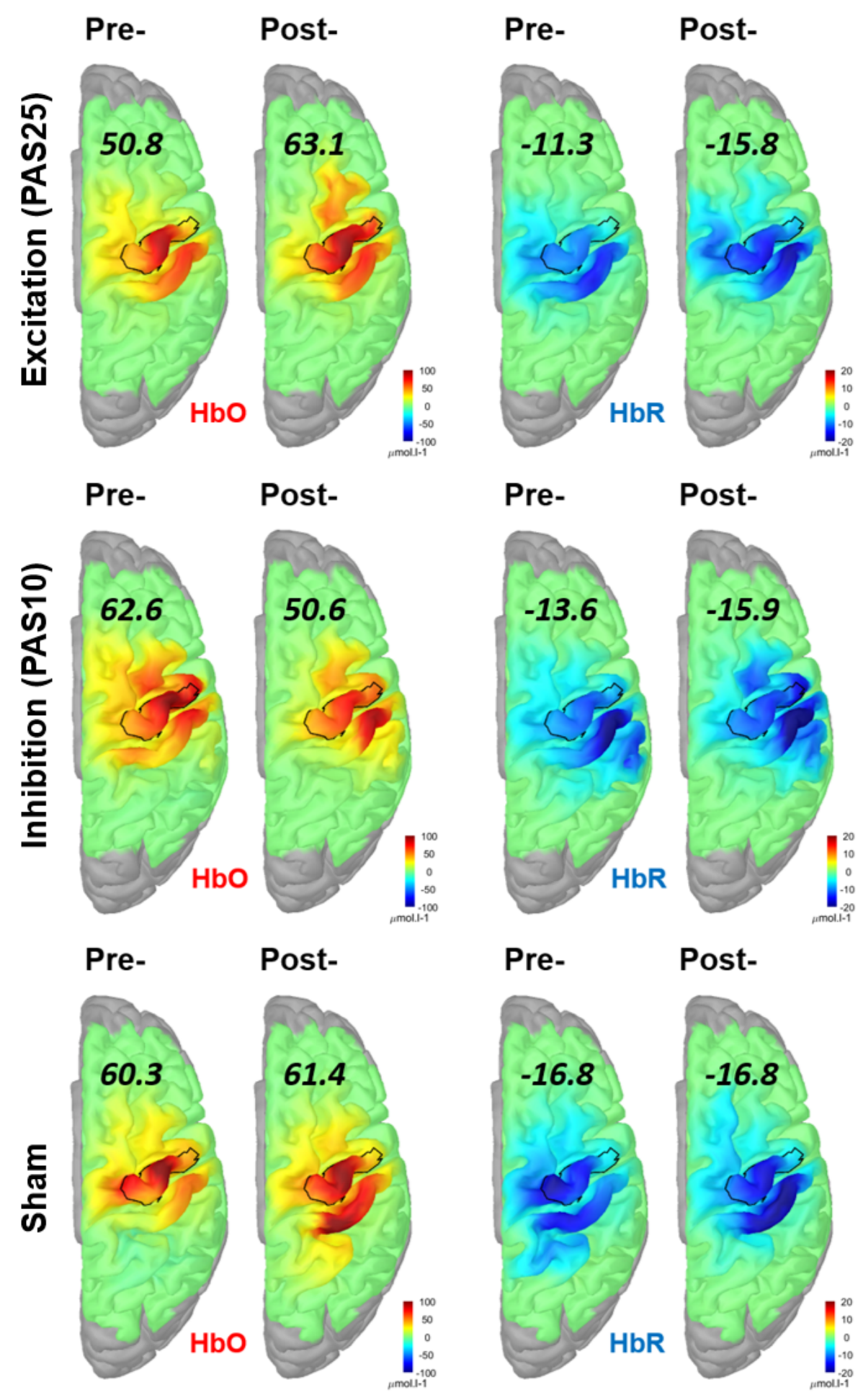

Fig.6. Group-level PAS effects on task-related hemodynamic responses. Group level reconstructed maps of $\mathrm{HbO}$ and $\mathrm{HbR}$ of each experimental session, pre- or post-, were shown in each row for PAS25, PAS10 and sham, respectively. Each map demonstrated the averaged corresponding individual-level taskrelated hemodynamic responses. Individual $\mathrm{HbO} / \mathrm{HbR}$ maps selected at their respective peak were first 
coregistered on the mid-surface of the MNI ICBM152 template using Freesurfer spherical registration and then averaged over all subjects. The numbers indicated on every map are reporting the mean $\mathrm{HbO}$ or $\mathrm{HbR}$ amplitude within the corresponding ROI. This group-level ROI was defined manually along the M1 cortex of the template cortical surface to cover the hand knob region. Note that these maps were mainly considered for the visualization of PAS effects, whereas the statistical summary of hemodynamic changes was extracted at the individual levels. Colour maps for pre- and post- maps were fixed for specific hemoglobin and session (e.g., HbO in PAS25). HbO amplitudes generally exhibit a larger range than $\mathrm{HbR}$ amplitudes.

\subsection{Relationship between PAS-related excitability and hemodynamic changes}

When pooling all sessions together (Fig.7a), the resulted linear regression between $\mathrm{HbO}$ ratio (HbR, respectively) and MEP ratio presented a positive relationship, but no significant association (MEP-HbO ratios, $r=0.19, \mathrm{p}=0.29$; MEP-HbR ratios, $r=0.18, \mathrm{p}=0.30$ ). Fig.7b illustrated the linear regression for sessions with concordant PAS effects (e.g., MEP and HbO and $\mathrm{HbR}$ ratios were simultaneously larger or smaller than 1), resulting in significant positive linear corrections between MEP ratio and both $\mathrm{HbO}(\mathrm{r}=0.82, \mathrm{p}<0.001)$ and $\mathrm{HbR}(\mathrm{r}=0.88$, $\mathrm{p}<0.001)$ ratios. No significant linear correlations were found when considering sessions with non-concordant PAS effects (Fig.7c). 

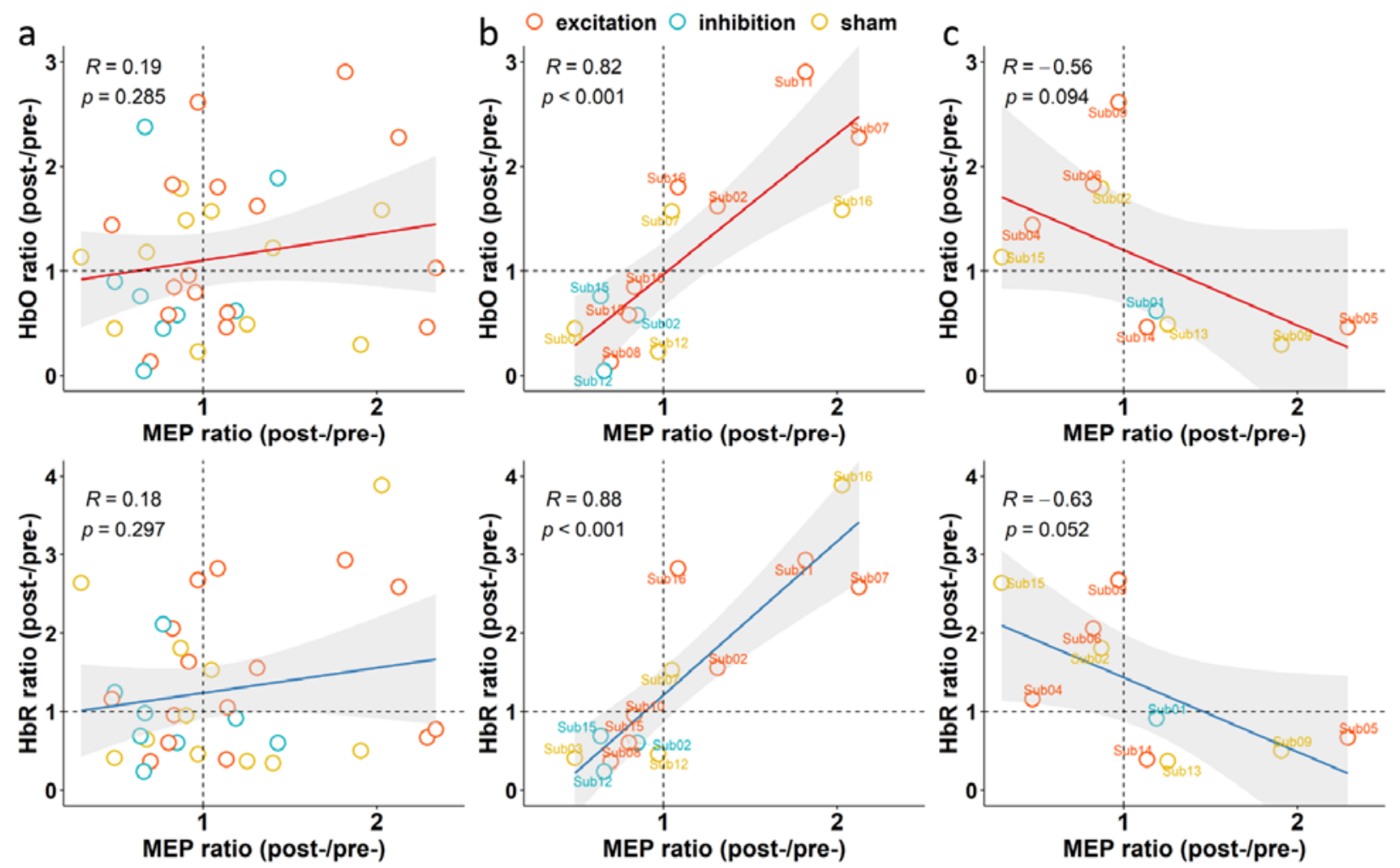

Fig.7. Correlates of task-related hemodynamic changes and PAS modulated excitability changes. The $1^{\text {st }}$ row presented $\mathrm{HbO}$ ratio as a function of MEP ratio and $2^{\text {nd }}$ row showed HbR ratio as a function of MEP ratio. Each point represented a session (e.g., PAS25 for Sub01, post-/pre- ratios). Sessions were color coded as red for PAS25, blue for PAS10 and yellow for sham. (a) linear regression between MEP ratios and $\mathrm{HbO} / \mathrm{HbR}$ ratios when considering all sessions. We found positive Pearson's correlations, $\mathrm{r}=0.19$ and $\mathrm{r}=0.18$ between MEP-HbO and MEP-HbR ratios, respectively, but none of them were statistically significant. (b) Linear regression for the PAS concordant sessions, in which MEP and HbO and $\mathrm{HbR}$ ratios were all larger than 1, or all smaller than 1. Both MEP-HbO and MEP-HbR cases demonstrated significant positive linear correlations ( $r=0.82$ and $r=0.88, p<0.001$ in both cases). Besides, both fitted lines were not far from the point $(1,1)$, e.g., $\mathrm{HbO}$ ratio $=0.96$ and $\mathrm{HbR}$ ratio $=1.16$, when fixing MEP ratio at 1 , which is consistent with our prior knowledge. (c) Linear regression for the PAS non-concordant cases acting as a control in which none of the correlations were statistically significant. Two lines were also slightly more distant from the point $(1,1)$, e.g., $\mathrm{HbO}$ ratio $=1.18$ and $\mathrm{HbR}$ ratio $=$ 1.43, when fixing MEP ratio at 1.

\section{Discussion}

Our study is the first one investigating the relationship between excitability and hemodynamic activity with simultaneous PAS-fNIRS in humans. We took advantage of this novel approach to 
achieve two main results: 1) fluctuations of cortical excitability were positively correlated with fluctuations of hemodynamic responses to the task; 2) there was a trend suggesting a positive linear relationship between effects of PAS on excitability and hemodynamic activity. Our results provide a unified view on two fundamental properties of cortical function. In addition, the demonstration of the effects of PAS on hemodynamic activity is relevant for the application of non-invasive brain stimulation techniques for the treatment of neuropsychiatric disorders. Finally, the tight link between excitability and hemodynamic activity may suggest that the effects on hemodynamics might be monitored via the standard spTMS technique.

\subsection{Correlation between cortical excitability and hemodynamic activity}

We demonstrated a link between excitability and task-related hemodynamic activity. Since PAS is known to induce variable effects across subjects, we pooled together all runs (before and after interventions) to investigate relationships between excitability and hemodynamic activity independently from specific PAS effects. Finger tapping is known to increase the metabolic demand and is therefore associated to increase $\mathrm{HbO}$, decrease $\mathrm{HbR}$ and increase blood volume [Kashou et al., 2016; Novi et al., 2020]. Moreover, finger tapping itself is known to increase cortical excitability [Koeneke et al., 2006]. The correlation that we found underlines that metabolic demands linked to finger tapping depend on the excitability state when the task was performed. In other words, the metabolic demands seem state-dependent, where brain state corresponded here to cortical excitability. State dependency is a very well-known concept in cortical function and involves multiple measures of neuronal activity such as activity, oscillations and connectivity [Giambattistelli et al., 2014; Gonçalves et al., 2006; Romei et al., 2008; Silvanto et al., 2008; Silvanto and Pascual-Leone, 2008].

\subsection{PAS effects on hemodynamic activity}

In the field of non-invasive human brain stimulation, two previous studies investigated the effect of M1 cortical excitability modulation on task-related hemodynamic activity. Kriváneková et al (2013) combined PAS and "offline" fMRI and reported no definite effects of PAS on either the task-related BOLD signal of the sensorimotor regions or resting-state functional connectivity. They reported that BOLD fluctuations following PAS were rather unpredictable, with almost no change after excitatory PAS, BOLD increase after inhibitory PAS and BOLD decrease after sham. Comparing these results to ours is challenging, but the low sampling frequency of fMRI 
might have contributed to their negative results. A low sampling rate means limited temporal sampling which might dilute small effects of excitability changes that we identified mostly around the peak of the hemodynamic response. Although PAS effects on $\mathrm{HbO}$ and $\mathrm{HbR}$ were also not significant in our study, we did find expected trends in some individuals and at the group level (Fig.4, 5 and 6). Our results are certainly in agreement with those reported by Chiang et al., (2007), who combined rTMS and fNIRS. They found that 1Hz rTMS over M1 induced an expected $\mathrm{HbO}$ increase in the contralateral cortex lasting up to 40 minutes, likely related to reciprocal inhibition mechanisms [Di Lazzaro et al., 2014]. Such cortical excitability effects on hemodynamic were also observed in other cortical regions. 5Hz rTMS on the right parietal cortex during the retention period of a match-to-sample task significantly increased $\mathrm{HbO}$ levels during the task period [Yamanaka et al., 2010]; whereas continuous theta-burst stimulation (cTBS) applied on the left dorsolateral prefrontal cortex (DLPFC) reduced Emotional Stroop task-evoked HbO levels bilaterally [Tupak et al., 2013]; cTBS on the right-DLPFC also reduced $\mathrm{HbO}$ during the dictator game [Maier et al., 2018].

Further development of our simultaneous fNIRS-TMS protocol may open new avenues for understanding the mechanism of PAS per se. For instance, the sparse rhythmic stimulation involved during PAS will allow investigating pulses-related hemodynamic effects and their build-up. Such analysis was out of our scope and will be considered in future investigations.

\subsection{Correlation between PAS effects on excitability and PAS effects on hemodynamic activity}

We also evaluated the correlation between excitability changes and hemodynamic activity changes modulated by interventions. Such analysis was challenging as the modeling involved dealing with the variabilities of MEP amplitudes, hemodynamic activity and PAS effects. Similar to other neuromodulatory techniques, PAS is known for inducing variable and sometimes unpredictable or even reversed effects [Suppa et al., 2017; Ziemann and Siebner, 2015], depending upon multiple factors, including state of the brain, genetic susceptibility, position of the coil, and much more [Ziemann and Siebner, 2015]. Using fMRI and PAS, Kriváneková et al., (2013) did not report any significant relationship between PAS related excitability changes and finger-tapping related BOLD changes. We found similar results regarding the correlation between MEP ratios and $\mathrm{HbO}(\mathrm{HbR}$, respectively) ratios when considering all sessions. Note that in our first correlation analysis we found that fluctuations of cortical excitability were positively 
correlated with fluctuations of hemodynamic responses to the task. Then if the variability of MEP amplitudes, hemodynamic activity and PAS effects do not introduce variability on ratio calculations of MEP and $\mathrm{HbO} / \mathrm{HbR}$, similar significant positive correlations should have been observed. Hence, we believe that these variabilities deflated the correlation value in our second correlation analysis. To further address this point, we constrained this correlation analysis for sessions with concordant (Fig.7b) or discordant (Fig.7c) PAS effects on MEP, HbO and HbR ratios. This proposed strategy simply regulates the influences of data variability on the correlation of ratios by claiming that the correlation should have existed in sessions where those three measurements varied in the same direction after PAS (i.e., less data variability). Interestingly, there was a significant correlation between $\mathrm{MEP}$ ratios and $\mathrm{HbO} / \mathrm{HbR}$ ratios. As a contrast, sessions with discordant PAS effects (Fig.7.c) did not show significant correlations. Moreover, when data exhibit less variability, $\mathrm{HbO}$ or $\mathrm{HbR}$ ratio should be close to 1 when fixing MEP ratio at 1 . In concordant case (Fig.7b), when fixing MEP ratio at 1 the fitted lines indicate that $\mathrm{HbO}$ ratio (0.96) and $\mathrm{HbR}$ ratio (1.16) were actually closer to 1 than in discordant case $(\mathrm{HbO}$ ratio $=1.18$ and $\mathrm{HbR}$ ratio $=1.43$, Fig. $7 \mathrm{c}$ ). Despite the simplicity of this regulation strategy using concordance, these evidences suggest that further advanced data analysis which better handles variability in the data may reveal a solid correlation between PAS effects on MEP and its effects on $\mathrm{HbO} / \mathrm{HbR}$. To summarize, our finding on this second correlation analysis underlines the complexity of non-invasive brain stimulation effects, which are often only investigated in the domain of excitability, but always involve also hemodynamic activity, electromagnetic activity, connectivity, and much more [Pellegrino et al., 2018; Pellegrino et al., 2019]. This finding also underlines the tight link between these cortical properties and may offer new opportunities for patients' treatment whenever the target action is a modulation of blood and hemoglobin supply.

\subsection{Reliability and robustness}

We did our best to acquire and analyze data in a robust way, including only male subjects, tuning PAS on individual N20, applying neuronavigation on individual MRI, collecting many MEPs (75 vs usual 20 trials). We considered personalized fNIRS data acquisition targeting the hand-knob using an optimal montage [Machado et al., 2014; Machado et al., 2018; Pellegrino et al., 2016] computed on the individual MRI. Physiological noise such as heartbeats, respiration and Mayer wave were minimized by filtering and regression using short-distance channels that only probe hemodynamics in the scalp [Zeff et al., 2007]. Optodes were positioned with neuronavigation 
and glued on head skin via collodion to ensure good contact and optimal probe design. We extracted $\mathrm{HbO} / \mathrm{HbR}$ features after 3D reconstructions along the cortical surface. fNIRS 3D reconstructions have been shown to provide more accurate quantification [Arridge, 1999; Boas et al., 2001] of $\mathrm{HbO} / \mathrm{HbR}$ than sensor level analyses applied in most fNIRS involved TMS studies [Curtin et al., 2019b; Curtin et al., 2019a; Oliviero et al., 1999; Thomson et al., 2011; Thomson et al., 2013]. More importantly, rigorous statistical procedures were conducted considering variabilities of data at different levels: 1) a bootstrap procedure to pool together data from all recordings instead of simply taking averages to investigate the relationship between excitability and hemodynamic activity; and 2) a resampling technique ensured to extract reliable, robust and data driven (intervention type blind) $\mathrm{HbO} / \mathrm{HbR}$ measures from fNIRS reconstructions. Finally, most of fNIRS involved TMS studies only reported results on HbO. However, we showed consensus results when considering both $\mathrm{HbO}$ and $\mathrm{HbR}$ signals, as recommended in a recent fNIRS guideline paper [Yücel et al., 2021].

\subsection{Limitations}

The small number of subjects and unbalanced data set are the main limitations of this study. However, considering the main contribution of this study was investigating the correlation, the number of sessions involved in the correlation analysis (Fig.3 and Fig.7) was sufficient. Secondly, the finger-tapping task and the spTMS session were not conducted at the same time, but within a few minutes. To be noted, applying TMS during the task would have answered different biological questions than the ones assessed here. Nonetheless, the time gap between TMS and motor tasks may have introduced some noise when investigating the correlation between $\mathrm{MEP}$ and $\mathrm{HbO} / \mathrm{HbR}$ because of the fluctuations of both measures over time. Finally, more advanced data analysis which better handles data variability may help to reveal a significant correlation between PAS effects on M1 excitability and its effects on task-related hemodynamic activity.

\section{Conclusions}

In conclusion, we demonstrated a linear relationship between brain excitability and task-related hemodynamic activity measured using personalized fNIRS. We also demonstrated that PAS may have effects on hemodynamic activity in addition to those on excitability and also influences the relationship between excitability and activity. These effects are not necessarily PAS-specific and 
may characterize other non-invasive brain stimulation techniques as well. Finally, our findings may further expand the field of non-invasive brain stimulation application for treating brain disorders by targeting those areas for which a modulation of hemodynamic activity is desired.

\section{Conflict of interest}

The authors declare no potential conflict of interest.

\section{Author contributions}

ZC: Formal analysis, Methodology, Data collection, Original draft preparation; Reviewing and Editing GP: Conceptualization, Investigation, Original draft preparation; Reviewing and Editing AS, ED, MU and CA: Data collection, Reviewing and Editing; JML: Methodology, Reviewing and Editing; SF: Conceptualization, Reviewing and Editing; CG: Conceptualization, Methodology, Supervision, Reviewing and Editing. 


\section{References}

Aarabi A, Osharina V, Wallois F (2017): Effect of confounding variables on hemodynamic response function estimation using averaging and deconvolution analysis: An event-related NIRS study. Neuroimage 155:25-49. http://dx.doi.org/10.1016/j.neuroimage.2017.04.048.

Ah Sen CB, Fassett HJ, El-Sayes J, Turco C V, Hameer MM, Nelson AJ (2017): Active and resting motor threshold are efficiently obtained with adaptive threshold hunting. PLoS One 12. https://doi.org/10.1371/journal.pone.0186007.

Arridge SR (1999): Optical tomography in medical imaging. Inverse Probl 15:R41. http://iopscience.iop.org/0266-5611/15/2/022\%5Cnpapers3://publication/doi/10.1088/0266$5611 / 15 / 2 / 022$.

Awiszus F, Feistner H, Urbach D, Bostock H (1999): Characterisation of paired-pulse transcranial magnetic stimulation conditions yielding intracortical inhibition or I-wave facilitation using a threshold-hunting paradigm. Exp Brain Res 129:317-324. https://pubmed.ncbi.nlm.nih.gov/10591905/.

Bandettini PA, Wong EC, Hinks RS, Tikofsky RS, Hyde JS (1992): Time course EPI of human brain function during task activation. Magn Reson Med 25:390-397. https://pubmed.ncbi.nlm.nih.gov/1614324/.

Boas DA, Gaudette T, Strangman G, Cheng X, Marota JJA, Mandeville JB (2001): The accuracy of near infrared spectroscopy and imaging during focal changes in cerebral hemodynamics. Neuroimage 13:76-90. http://linkinghub.elsevier.com/retrieve/pii/S1053811900906748.

Cai Z, Machado A, Chowdhury RA, Spilkin A, Vincent T, Aydin U, Pellegrino G, Lina J-M, Grova C (2021a): Diffuse optical reconstructions of NIRS data using Maximum Entropy on the Mean. bioRxiv:2021.02.22.432263. https://doi.org/10.1101/2021.02.22.432263.

Cai Z, Uji M, Aydin Ü, Pellegrino G, Spilkin A, Delaire É, Abdallah C, Lina J, Grova C (2021b): Evaluation of a personalized functional near infra-red optical tomography workflow using maximum entropy on the mean. Hum Brain Mapp:hbm.25566. https://onlinelibrary.wiley.com/doi/10.1002/hbm.25566.

Carson RG, Kennedy NC (2013): Modulation of human corticospinal excitability by paired 
associative stimulation. Front Hum Neurosci 7:1-28.

http://journal.frontiersin.org/article/10.3389/fnhum.2013.00823/abstract.

Chiang TC, Vaithianathan T, Leung T, Lavidor M, Walsh V, Delpy DT (2007): Elevated

haemoglobin levels in the motor cortex following $1 \mathrm{~Hz}$ transcranial magnetic stimulation: A preliminary study. Exp Brain Res 181:555-560.

Curtin A, Ayaz H, Tang Y, Sun J, Wang J, Tong S (2019a): Enhancing neural efficiency of cognitive processing speed via training and neurostimulation: An fNIRS and TMS study. Neuroimage 198:73-82. https://doi.org/10.1016/j.neuroimage.2019.05.020.

Curtin A, Tong S, Sun J, Wang J, Onaral B, Ayaz H (2019b): A systematic review of integrated functional near-infrared spectroscopy (fNIRS) and transcranial magnetic stimulation (TMS) studies. Front Neurosci 13.

Fischl B, Salat DH, Busa E, Albert M, Dieterich M, Haselgrove C, Van Der Kouwe A, Killiany R, Kennedy D, Klaveness S, Montillo A, Makris N, Rosen B, Dale AM (2002): Whole brain segmentation: Automated labeling of neuroanatomical structures in the human brain. Neuron 33:341-355.

http://eutils.ncbi.nlm.nih.gov/entrez/eutils/elink.fcgi?dbfrom=pubmed\&id=11832223\&retm ode=ref\&cmd=prlinks\%5Cnpapers2://publication/uuid/23BE8334-219B-4E46-AE68C9D02B8AC109.

Fonov V, Evans AC, Botteron K, Almli CR, McKinstry RC, Collins DL (2011): Unbiased average age-appropriate atlases for pediatric studies. Neuroimage 54:313-327.

Fonov V, Evans A, McKinstry R, Almli C, Collins D (2009): Unbiased nonlinear average ageappropriate brain templates from birth to adulthood. Neuroimage 47:S102.

Giambattistelli F, Tomasevic L, Pellegrino G, Porcaro C, Melgari JM, Rossini PM, Tecchio F (2014): The spontaneous fluctuation of the excitability of a single node modulates the internodes connectivity: A TMS-EEG study. Hum Brain Mapp 35:1740-1749. https://pubmed.ncbi.nlm.nih.gov/23670997/.

Glover GH (2011): Overview of functional magnetic resonance imaging. Neurosurgery Clinics of North America. NIH Public Access. /pmc/articles/PMC3073717/?report=abstract.

Gonçalves SI, De Munck JC, Pouwels PJW, Schoonhoven R, Kuijer JPA, Maurits NM, 
Hoogduin JM, Van Someren EJW, Heethaar RM, Lopes Da Silva FH (2006): Correlating the alpha rhythm to BOLD using simultaneous EEG/fMRI: Inter-subject variability. Neuroimage 30:203-213. https://pubmed.ncbi.nlm.nih.gov/16290018/.

Gow D, Rothwell J, Hobson A, Thompson D, Hamdy S (2004): Induction of long-term plasticity in human swallowing motor cortex following repetitive cortical stimulation. Clin Neurophysiol 115:1044-1051.

Gramigna V, Pellegrino G, Cerasa A, Cutini S, Vasta R, Olivadese G, Martino I, Quattrone A (2017): Near-Infrared Spectroscopy in Gait Disorders: Is It Time to Begin?

Neurorehabilitation and Neural Repair. https://doi.org/10.1177/1545968317693304.

Gregg NM, White BR, Zeff BW, Berger AJ, Culver JP (2010): Brain specificity of diffuse optical imaging: improvements from superficial signal regression and tomography. Front Neuroenergetics 2. http://www.ncbi.nlm.nih.gov/pubmed/20725524.

Hallett M, Di Iorio R, Rossini PM, Park JE, Chen R, Celnik P, Strafella AP, Matsumoto H, Ugawa Y (2017): Contribution of transcranial magnetic stimulation to assessment of brain connectivity and networks. Clin Neurophysiol 128:2125-2139. https://doi.org/10.1016/j.clinph.2017.08.007.

Hattemer K, Knake S, Reis J, Rochon J, Oertel WH, Rosenow F, Hamer HM (2007): Excitability of the motor cortex during ovulatory and anovulatory cycles: A transcranial magnetic stimulation study. Clin Endocrinol (Oxf) 66:387-393. https://pubmed.ncbi.nlm.nih.gov/17302873/.

Jöbsis FF (1977): Noninvasive, infrared monitoring of cerebral and myocardial oxygen sufficiency and circulatory parameters. Science (80- ) 198:1264-1266. https://pubmed.ncbi.nlm.nih.gov/929199/.

Kashou NH, Giacherio BM, Nahhas RW, Jadcherla SR (2016): Hand-grasping and finger tapping induced similar functional near-infrared spectroscopy cortical responses. Neurophotonics 3:025006.

Koeneke S, Lutz K, Herwig U, Ziemann U, Jäncke L (2006): Extensive training of elementary finger tapping movements changes the pattern of motor cortex excitability. Exp Brain Res 174:199-209. https://pubmed.ncbi.nlm.nih.gov/16604315/. 
Kriváneková L, Baudrexel S, Bliem B, Ziemann U (2013): Relation of brain stimulation induced changes in MEP amplitude and BOLD signal. Brain Stimul 6:330-339. https://www.sciencedirect.com/science/article/pii/S1935861X12001003?via\%3Dihub\#fig3.

Kwong KK, Belliveau JW, Chesler DA, Goldberg IE, Weisskoff RM, Poncelet BP, Kennedy DN, Hoppel BE, Cohen MS, Turner R, Cheng - HM, Brady TJ, Rosen BR (1992): Dynamic magnetic resonance imaging of human brain activity during primary sensory stimulation. Proc Natl Acad Sci U S A 89:5675-5679. https://pubmed.ncbi.nlm.nih.gov/1608978/.

Di Lazzaro V, Dileone M, Capone F, Pellegrino G, Ranieri F, Musumeci G, Florio L, Di Pino G, Fregni F (2014): Immediate and late modulation of interhemipheric imbalance with bilateral transcranial direct current stimulation in acute stroke. Brain Stimul 7:841-848. https://pubmed.ncbi.nlm.nih.gov/25458712/.

Lee JC, Croarkin PE, Ameis SH, Sun Y, Blumberger DM, Rajji TK, Daskalakis ZJ (2017): Paired-Associative Stimulation-Induced Long-term Potentiation-Like Motor Cortex Plasticity in Healthy Adolescents. Front Psychiatry 8:1-8. http://journal.frontiersin.org/article/10.3389/fpsyt.2017.00095/full.

Levy WB, Steward O (1983): Temporal contiguity requirements for long-term associative potentiation/depression in the hippocampus. Neuroscience 8:791-797. https://linkinghub.elsevier.com/retrieve/pii/0306452283900106.

Loo CK, Taylor JL, Gandevia SC, McDarmont BN, Mitchell PB, Sachdev PS (2000): Transcranial magnetic stimulation (TMS) in controlled treatment studies: Are some "sham" forms active? Biol Psychiatry 47:325-331.

Machado A, Cai Z, Pellegrino G, Marcotte O, Vincent T, Lina J-M, Kobayashi E, Grova C (2018): Optimal positioning of optodes on the scalp for personalized functional nearinfrared spectroscopy investigations. J Neurosci Methods 309:91-108. https://www.sciencedirect.com/science/article/pii/S0165027018302425?via\%3Dihub.

Machado A, Cai Z, Vincent T, Pellegrino G, Lina JM, Kobayashi E, Grova C (2021):

Deconvolution of hemodynamic responses along the cortical surface using personalized functional near infrared spectroscopy. Sci Rep:1-19. https://doi.org/10.1038/s41598-02185386-0. 
Machado A, Marcotte O, Lina JM, Kobayashi E, Grova C (2014): Optimal optode montage on electroencephalography/functional near-infrared spectroscopy caps dedicated to study epileptic discharges. J Biomed Opt 19:026010.

http://biomedicaloptics.spiedigitallibrary.org/article.aspx?doi=10.1117/1.JBO.19.2.026010.

Maier MJ, Rosenbaum D, Haeussinger FB, Brüne M, Enzi B, Plewnia C, Fallgatter AJ, Ehlis AC (2018): Forgiveness and cognitive control - Provoking revenge via theta-burst-stimulation of the DLPFC. Neuroimage 183:769-775.

Michou E, Mistry S, Jefferson S, Tyrrell P, Hamdy S (2014): Characterizing the mechanisms of central and peripheral forms of neurostimulation in chronic dysphagic stroke patients. Brain Stimul 7:66-73.

Navarro de Lara LI, Tik M, Woletz M, Frass-Kriegl R, Moser E, Laistler E, Windischberger C (2017): High-sensitivity TMS/fMRI of the Human Motor Cortex Using a Dedicated Multichannel MR Coil. Neuroimage 150:262-269. http://dx.doi.org/10.1016/j.neuroimage.2017.02.062.

Navarro De Lara LI, Windischberger C, Kuehne A, Woletz M, Sieg J, Bestmann S, Weiskopf N, Strasser B, Moser E, Laistler E (2015): A novel coil array for combined TMS/fMRI experiments at 3 T. Magn Reson Med 74:1492-1501.

Novi SL, Forero EJ, Rubianes Silva JAI, de Souza NGSR, Martins GG, Quiroga A, Wu ST, Mesquita RC (2020): Integration of Spatial Information Increases Reproducibility in Functional Near-Infrared Spectroscopy. Front Neurosci 14:1-12.

Oliviero A, Di Lazzaro V, Piazza O, Profice P, Pennisi MA, Delia Corte F, Tonali P (1999): Cerebral blood flow and metabolic changes produced by repetitive magnetic brain stimulation. J Neurol 246:1164-1168.

Parks NA (2013): Concurrent application of TMS and near-infrared optical imaging: methodological considerations and potential artifacts. Front Hum Neurosci 7:592. http://journal.frontiersin.org/article/10.3389/fnhum.2013.00592/abstract.

Pellegrino G, Arcara G, Di Pino G, Turco C, Maran M, Weis L, Piccione F, Siebner HR (2019): Transcranial direct current stimulation over the sensory-motor regions inhibits gamma synchrony. Hum Brain Mapp 40:2736-2746. 
Pellegrino G, Machado A, von Ellenrieder N, Watanabe S, Hall JA, Lina JM, Kobayashi E, Grova C (2016): Hemodynamic response to interictal epileptiform discharges addressed by personalized EEG-fNIRS recordings. Front Neurosci 10:102.

http://www.ncbi.nlm.nih.gov/pubmed/27047325.

Pellegrino G, Maran M, Turco C, Weis L, Di Pino G, Piccione F, Arcara G (2018): Bilateral Transcranial Direct Current Stimulation Reshapes Resting-State Brain Networks: A Magnetoencephalography Assessment. Neural Plast 2018.

R Core Team (2020): R: A Language and Environment for Statistical Computing. Vienna, Austria. http://www.r-project.org/.

Raffin E, Pellegrino G, Di Lazzaro V, Thielscher A, Siebner HR (2015): Bringing transcranial mapping into shape: Sulcus-aligned mapping captures motor somatotopy in human primary motor hand area. Neuroimage 120:164-175.

Romei V, Brodbeck V, Michel C, Amedi A, Pascual-Leone A, Thut G (2008): Spontaneous fluctuations in posterior $\alpha$-band EEG activity reflect variability in excitability of human visual areas. Cereb Cortex 18:2010-2018. https://pubmed.ncbi.nlm.nih.gov/18093905/.

Rossi S, Hallett M, Rossini PM, Pascual-Leone A, Avanzini G, Bestmann S, Berardelli A, Brewer C, Canli T, Cantello R, Chen R, Classen J, Demitrack M, Di Lazzaro V, Epstein CM, George MS, Fregni F, Ilmoniemi R, Jalinous R, Karp B, Lefaucheur JP, Lisanby S, Meunier S, Miniussi C, Miranda P, Padberg F, Paulus W, Peterchev A, Porteri C, Provost M, Quartarone A, Rotenberg A, Rothwell J, Ruohonen J, Siebner H, Thut G, Valls-Solè J, Walsh V, Ugawa Y, Zangen A, Ziemann U (2009): Safety, ethical considerations, and application guidelines for the use of transcranial magnetic stimulation in clinical practice and research. Clinical Neurophysiology. Elsevier.

Rossini PM, Burke D, Chen R, Cohen LG, Daskalakis Z, Di Iorio R, Di Lazzaro V, Ferreri F, Fitzgerald PB, George MS, Hallett M, Lefaucheur JP, Langguth B, Matsumoto H, Miniussi C, Nitsche MA, Pascual-Leone A, Paulus W, Rossi S, Rothwell JC, Siebner HR, Ugawa Y, Walsh V, Ziemann U (2015): Non-invasive electrical and magnetic stimulation of the brain, spinal cord, roots and peripheral nerves: Basic principles and procedures for routine clinical and research application: An updated report from an I.F.C.N. Committee. Clin 
Neurophysiol 126:1071-1107. http://dx.doi.org/10.1016/j.clinph.2015.02.001.

Scholkmann F, Kleiser S, Metz AJ, Zimmermann R, Mata Pavia J, Wolf U, Wolf M (2014): A

review on continuous wave functional near-infrared spectroscopy and imaging instrumentation and methodology. NeuroImage.

Siebner HR, Bergmann TO, Bestmann S, Massimini M, Johansen-Berg H, Mochizuki H, Bohning DE, Boorman ED, Groppa S, Miniussi C, Pascual-Leone A, Huber R, Taylor PCJ, Ilmoniemi RJ, De Gennaro L, Strafella AP, Kähkönen S, Klöppel S, Frisoni GB, George MS, Hallett M, Brandt SA, Rushworth MF, Ziemann U, Rothwell JC, Ward N, Cohen LG, Baudewig J, Paus T, Ugawa Y, Rossini PM (2009): Consensus paper: Combining transcranial stimulation with neuroimaging. Brain Stimul 2:58-80.

Silvanto J, Muggleton N, Walsh V (2008): State-dependency in brain stimulation studies of perception and cognition. Trends Cogn Sci 12:447-454. https://pubmed.ncbi.nlm.nih.gov/18951833/.

Silvanto J, Pascual-Leone A (2008): State-dependency of transcranial magnetic stimulation. Brain Topography. Brain Topogr. https://pubmed.ncbi.nlm.nih.gov/18791818/.

Stefan K (2000): Induction of plasticity in the human motor cortex by paired associative stimulation. Brain 123:572-584. https://academic.oup.com/brain/articlelookup/doi/10.1093/brain/123.3.572.

Suppa A, Quartarone A, Siebner H, Chen R, Di Lazzaro V, Del Giudice P, Paulus W, Rothwell JC, Ziemann U, Classen J (2017): The associative brain at work: Evidence from paired associative stimulation studies in humans. Clin Neurophysiol 128:2140-2164. https://doi.org/10.1016/j.clinph.2017.08.003.

Tadel F, Baillet S, Mosher JC, Pantazis D, Leahy RM (2011): Brainstorm: A user-friendly application for MEG/EEG analysis. Comput Intell Neurosci 2011:879716. http://www.ncbi.nlm.nih.gov/pubmed/21584256.

Thomson RH, Cleve TJ, Bailey NW, Rogasch NC, Maller JJ, Daskalakis ZJ, Fitzgerald PB (2013): Blood oxygenation changes modulated by coil orientation during prefrontal transcranial magnetic stimulation. Brain Stimul 6:576-581. https://ac.elscdn.com/S1935861X12002070/1-s2.0-S1935861X12002070-main.pdf?_tid=79f626c2- 
bd7d-11e7-959d-0000aacb35f\&acdnat=1509373409_f1e2e340d0a3f9b39846dfea9e5bbdee.

Thomson RH, Maller JJ, Daskalakis ZJ, Fitzgerald PB (2011): Blood oxygenation changes resulting from suprathreshold transcranial magnetic stimulation. Brain Stimul 4:165-168. https://ac.els-cdn.com/S1935861X10001622/1-s2.0-S1935861X10001622main.pdf?_tid=81c5395c-bd8b-11e7-a91d00000aab0f26\&acdnat=1509379437_7cf18aa5d3313455bb1980c40b90c90c.

Tupak S V., Dresler T, Badewien M, Hahn T, Ernst LH, Herrmann MJ, Deckert J, Ehlis AC, Fallgatter AJ (2013): Inhibitory transcranial magnetic theta burst stimulation attenuates prefrontal cortex oxygenation. Hum Brain Mapp 34:150-157.

Vasta R, Cerasa A, Gramigna V, Augimeri A, Olivadese G, Pellegrino G, Martino I, Machado A, Cai Z, Caracciolo M, Grova C, Quattrone A (2017): The movement time analyser task investigated with functional near infrared spectroscopy: an ecologic approach for measuring hemodynamic response in the motor system. Aging Clin Exp Res 29:311-318.

Wang WT, Xu B, Butman JA (2017): Improved SNR for combined TMS-fMRI: A support device for commercially available body array coil. J Neurosci Methods 289:1-7. http://dx.doi.org/10.1016/j.jneumeth.2017.06.020.

Yamanaka K, Yamagata B, Tomioka H, Kawasaki S, Mimura M (2010): Transcranial magnetic stimulation of the parietal cortex facilitates spatial working memory: Near-infrared spectroscopy study. Cereb Cortex 20:1037-1045.

Yücel MA, Lühmann A v., Scholkmann F, Gervain J, Dan I, Ayaz H, Boas D, Cooper RJ, Culver J, Elwell CE, Eggebrecht A, Franceschini MA, Grova C, Homae F, Lesage F, Obrig H, Tachtsidis I, Tak S, Tong Y, Torricelli A, Wabnitz H, Wolf M (2021): Best practices for fNIRS publications. Neurophotonics 8:1-34.

Zangrandi A, Mioli A, D’Alonzo M, Formica D, Pellegrino G, Di Pino G (2019): Conditioning transcranial magnetic stimulation of ventral premotor cortex shortens simple reaction time. Cortex 121:322-331.

Zeff BW, White BR, Dehghani H, Schlaggar BL, Culver JP (2007): Retinotopic mapping of adult human visual cortex with high-density diffuse optical tomography. Proc Natl Acad Sci 104:12169-12174. http://www.pnas.org/cgi/doi/10.1073/pnas.0611266104. 
bioRxiv preprint doi: https://doi.org/10.1101/2021.09.29.462418; this version posted October 1, 2021. The copyright holder for this preprint

(which was not certified by peer review) is the author/funder, who has granted bioRxiv a license to display the preprint in perpetuity. It is made available under aCC-BY-NC-ND 4.0 International license.

Ziemann U, Siebner HR (2015): Inter-subject and intersession variability of plasticity induction by non-invasive brain stimulation: Boon or bane? Brain Stimulation. Elsevier Inc. 


\section{Appendices}

\section{A: Experiment design and Data acquisition}

\section{Anatomical MRI}

To guide TMS procedure and to calculate the head model for fNIRS acquisitions and analyses, MR brain images were acquired for each participant, using a General Electric Discovery MR750 3T scanner at the PERFORM Center of Concordia University, Montréal, Canada. T1-weighted images were acquired using the 3D BRAVO sequence and the following parameters: $1 \times 1 \times 1$ $\mathrm{mm}^{3}$, 192 axial slices, $256 \times 256$ matrix. T2-weighted anatomical images were acquired using the 3D Cube T2 sequence and the following parameters: $1 \times 1 \times 1 \mathrm{~mm}^{3}$ voxels, 168 sagittal slices, $256 \times 256$ matrix.

\section{fNIRS data acquisition}

fNIRS data were acquired using a Brainsight fNIRS machine (Rogue-Research Inc, Montréal, Canada) sampling at $10 \mathrm{~Hz}$. A neuro-navigation system (Rogue-Research Inc, Montréal) with individual MRI images guided the installation of the optodes according to the previously estimated optimal montage (see Fig.2). Optodes were glued over the subject's scalp with collodion, taking care to remove the hair between skin and sensor, thus allowing reducing motion artifacts during prolonged recordings (Fig.2) [Machado et al., 2018; Pellegrino et al., 2016; Yücel et al., 2014].

\section{Measurement of M1 cortical excitability}

TMS was delivered with a Magstim $200^{2}$ stimulator (Magstim Company, Carmarthenshire, Wales, UK) connected to a figure-8 coil (Magstim double 70mm remote control coil). Subjects were sitting in a comfortable armchair with the support of the left arm and neck (see Fig.2.d). To reduce potential motion, we placed the head of the participant between a mechanical arm wrapped with a soft cushion and the TMS coil (see Fig.2.d). TMS procedures were guided by the neuro-navigation system Brainsight (Rogue-Research Inc, Montréal, Canada). TMS coil was placed tangential to the scalp and with a $45^{\circ}$ angle to the midline of the head [Thomson et al., 2013]. MEPs were recorded by a BrainAmp ExG bipolar system with 2 TECA disposable 20mm disk electromyography (EMG) electrodes attached on the right abductor pollicis brevis (APB), 
with a standard belly-tendon montage (Fig2.d). The TMS "hot spot" was found for each session as the location with the maximal Motor Evoked Potentials (MEP). Resting motor threshold (RMT) was defined efficiently using the TMS Motor Threshold Assessment Tool (MTAT 2.0, http://www.clinicalresearcher.org/software.htm) based on maximum-likelihood parameter estimation by sequential testing approach [Ah Sen et al., 2017; Awiszus et al., 1999].

\section{Somatosensory evoked potentials}

Electrical stimulation (Digitimer DS7A, Letchworth Garden City, U.K) at the left wrist (e.g., Median Nerve) was performed after the MRI scan. Two bipolar EEG (BrainAmp ExG, Brain Products $\mathrm{GmbH}$, Germany) electrodes located at $\mathrm{CP} 3$ and $\mathrm{CP} 4$ were used to measure subject specific N20 latency. Stimulation was delivered slightly above the motor threshold at $4 \mathrm{~Hz}$ for 2 minutes. N20 latency was visually estimated via the online segment averaging functionality of the BrainVision Recorder (BrainAmp ExG, Brain Products GmbH, Germany).

\section{B: Reliable and robust estimation of task-related hemodynamic responses using fNIRS 3D reconstructions and resampling technique}

To conduct a reliable and robust task-related hemodynamic response estimation to compare hemodynamics before and after interventions, we combined fNIRS 3D reconstruction and resampling techniques to appropriately handle variability between trials and influence of eventual motion artifacts. The workflow consisted of 3 steps: a) resampling of "all the possible" averaged optical density time courses; b) fNIRS 3D reconstruction along the cortex using the MEM and c) definition of a session specific spatial ROI for $\mathrm{HbO} / \mathrm{HbR}$ features extraction.

a) We conducted a resampling of "all the possible" averaged optical density time course (-10s to 30s) as the input for further fNIRS 3D reconstructions. First, for every 20 blocks of preprocessed optical density data, we sub-averaged all 16 out of 20 blocks, resulting in a total of $C_{20}^{16}=4845$ possible combinations. These averaged trials were then sorted by the averaged signal-to-noise ratio (SNR) for two wavelengths. SNR was calculated by the largest amplitude among all channels from 0s to 30s, normalized by the mean of standard deviation over all channels during the baseline [-10s, 0s]. Finally, we selected 101 out of 4845 sub-averaged blocks centred around the median SNR of all sub-averages. The selection of 16 trials for sub-averaging was empirically defined according to the observation that 
usually there were around 4 artifacts contaminated blocks per condition (i.e., containing eventual motion artifacts). For artifacts contaminated data, large motion artifacts would then result in large SNR of sub-averaged trials. On the other hand, for data that are not contaminated by artifacts, the SNR distribution will be flat. Therefore, selecting subaveraged trials around the median SNR should ensure a good representation of fNIRS responses, discarding artifact sub-averages. We finally chose 101 sub-averaged trials to ensure a good representation of the underlying distribution of SNR values, while being sensitive to the inherent variability of task-evoked fNIRS responses.

b) fNIRS 3D reconstruction along the cortex was conducted using the MEM method applied to each of 101 sub-averaged trials specific for task run (e.g., pre-PAS25 of Sub01). We, therefore, took into account the variability of the hemodynamic response, instead of considering only one averaged response. "All the possible" spatiotemporal maps of $\mathrm{HbO}$ and HbR along the cortical surface were reconstructed using MEM from these 101 resampled sub-averaged trials. MEM is an efficient nonlinear probabilistic Bayesian framework to incorporate prior knowledge in the solution of the inverse problem for 3D reconstruction [Amblard et al., 2004]. We have demonstrated the excellent accuracy of MEM, especially its high sensitivity and specificity to the spatial extent of the underlying generators in the context of electro-/magneto-encephalogram source imaging [Chowdhury et al., 2013; Chowdhury et al., 2016; Grova et al., 2016; Hedrich et al., 2017; Heers et al., 2016; Pellegrino et al., 2020] as well as for fNIRS 3D reconstructions [Cai et al., 2021].

c) A session specific (e.g., PAS25 of Sub01) spatial ROI was finally defined along the cortical surface, to extract the reconstructed $\mathrm{HbO} / \mathrm{HbR}$ time courses features from MEM reconstructed spatiotemporal maps. To do so, we first extracted the task run specific (e.g., pre-PAS25 of Sub01) $\mathrm{HbO} / \mathrm{HbR}$ peak maps, at the peak timing estimated from the reconstructed time courses within the hand knob. This resulted in $101 \mathrm{HbO} / \mathrm{HbR}$ peak spatial maps for each task run. Then, a one-sample t-test of $\mathrm{HbO}$ (respectively $\mathrm{HbR}$ ) amplitude for each vertex of the map was performed across 101 maps for each task run. Regions exhibiting a significant response $(\mathrm{p}<0.05$, false discovery rate corrected) were kept as the cortical area, which contained significant hemodynamic responses of one task run. For each session (e.g., PAS25 of Sub01), this analysis resulted in 4 regions exhibiting significant hemodynamic responses, i.e., $\mathrm{HbO}$ and $\mathrm{HbR}$ response t-maps, in pre- and post-intervention conditions. The 
final ROI was defined as the intersection between these 4 statistically significant regions to ensure reliability and robustness. Note that this final ROI was confirmed to be within the M1 cortical area for all participants. Two PAS10 sessions and 1 sham session were rejected due to the empty intersection regions (no overlapping between the 4 regions), suggesting a lack of reliability of the resulting hemodynamic responses. We defined this ROI for each intervention session rather than for each subject, considering the variability of task performance within each subject, since the intervention sessions of each subject were performed on different days.

\section{C: PAS effects on cortical excitability and hemodynamic activity}

As shown in Fig.C1, PAS exerted the expected group level effect with average MEP ratios (mean \pm SEM) of $1.23 \pm 0.15,0.88 \pm 0.08$ and $1.08 \pm 0.15$ for PAS25, PAS10 and Sham, respectively. Such group level trends were also manifested in $\mathrm{HbO}$ and $\mathrm{HbR}$ ratios. For instance, the group level effect with average $\mathrm{HbO}$ ratios (mean \pm SEM) were 1.27 $\pm 0.21,0.95 \pm 0.24$ and $1.04 \pm 0.17$ for PAS25, PAS10 and Sham, respectively; and the group level effect with average HbR ratios (mean \pm SEM) were $1.48 \pm 0.24,1.02 \pm 0.20$ and $1.23 \pm 0.35$ for PAS25, PAS10 and Sham, respectively. However, these effects did not reach statistical significance (one-sample t-test against $1, \mathrm{p}>0.05$ ). There were also no significant differences among 3 interventions (ANOVA test $\mathrm{p}>0.05)$.
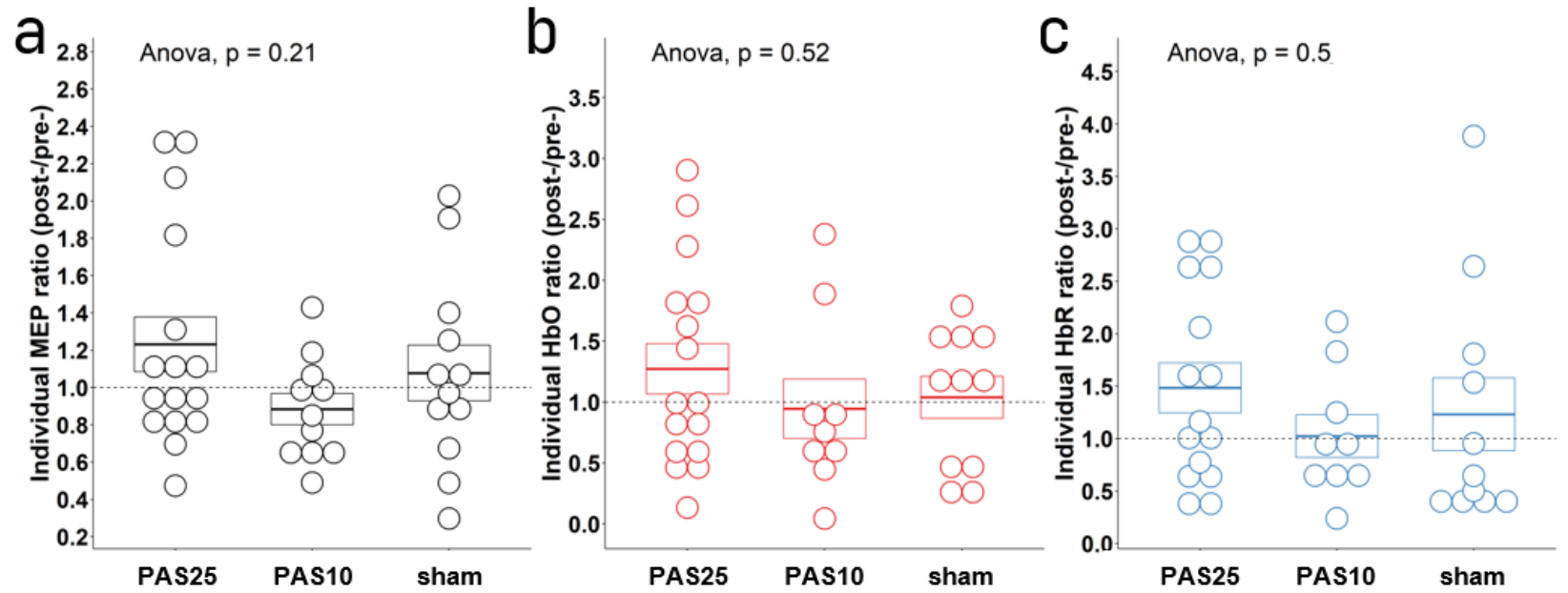

Fig.C1. PAS effects on cortical excitability and hemodynamic activity. At the group level, Mean \pm SEM (standard error of the mean) for the excitatory, inhibitory and sham session were: (a) $1.23 \pm 0.15,0.88 \pm 0.08$ and $1.08 \pm 0.15$ for MEP ratios; (b) $1.27 \pm 0.21,0.95 \pm 0.24$ and $1.04 \pm 0.17$ for $\mathrm{HbO}$ 
ratios; and (c) $1.48 \pm 0.24,1.02 \pm 0.20$ and $1.23 \pm 0.35$ for HbR ratios. Even if trends were observed, none of these ratios were significantly different from 1 based on a one-sample t-test. The individual ratios represented by each dot demonstrated a relatively large variance of the ratios for each scenario. Boxes showed the Mean \pm SEM in each case. Note that one MEP session (e.g., Sub16, PAS10) was rejected from the whole analysis due to a high ratio of 2.81; and one HbR session (e.g., Sub12, PAS25, HbR) was rejected from the whole analysis due to a high ratio of 5.45. A similar process was also considered in Kriváneková et al (2013). 